\title{
Augmented projections for ptychographic imaging
}

\author{
Stefano Marchesini and Andre Schirotzek \\ Advanced Light Source, Lawrence Berkeley National Laboratory, Berkeley, CA 94720* \\ Chao Yang \\ Computational Research Division, Lawrence Berkeley National Laboratory, Berkeley, CA 94720.' \\ Hau-tieng $\mathrm{Wu}$ \\ Statistics, University of California, Berkeley, CA 94720 \\ Filipe Maia \\ NERSC, Lawrence Berkeley National Laboratory, Berkeley, CA 94720

\begin{abstract}
Ptychography is a popular technique to achieve diffraction limited resolution images of a two or three dimensional sample using high frame rate detectors. We introduce a relaxation of common projection algorithms to account for instabilities given by intensity and background fluctuations, position errors, or poor calibration using multiplexing illumination. This relaxation introduces an additional phasing optimization at every step that enhances the convergence rate of common projection algorithms. Numerical tests exhibit the exact recovery of the object and the perturbations when there is high redundancy in the data.
\end{abstract}

\section{INTRODUCTION}

Ptychography was proposed in 1969 to improve the resolution in electron or x-ray microscopy ${ }^{1-4}$. In a scanning microscope, a small beam is focused onto the sample via a lens, and the transmission is measured in a singleelement detector. The image is built up by plotting the transmission as a function of the sample position as it is rastered across the beam. In such microscope, the resolution of the image is given by the beam size. In ptychography, one replaces the single element detector with a two dimensional array detector such as a CCD and measures the intensity distribution at many scattering angles. Each recorded diffraction pattern contains shortspatial Fourier frequency information about features that are smaller than the beam-size, enabling higher resolution. At short wavelengths however it is only possible to measure the intensity of the diffracted light. To reconstruct an image of the object, one needs to retrieve the phase information. With measured amplitude and phase information, a high resolution image can be readily computed, phase contrast imaging becomes possible, and the depth of focus for 3D micro-tomography is no longer a problem. While phase retrieval problems are notoriously difficult to solve numerically, the problem is made tractable in ptychography by using redundant measurements. In practice, multiple views of the same region of the object are recorded by using a small step size-relative to the size of the illuminating beam- when scanning the sample. With high speed detectors ${ }^{5,6}$ and ever brighter light sources, ptychographic imaging is becoming increasingly popular.

A practical issue in ptychographic reconstruction are the strict requirements of the experimental geometry to achieve high quality data. For example, the need for stable, well controlled coherent illumination of the sample, limited detector speed and response function all contribute to limit the specifications of a ptychographic microscope. New methods to work with unknown illuminations were proposed ${ }^{7-10}$. They are now used to calibrate high quality $\mathrm{x}$-ray optics ${ }^{11-13}$ and space telescopes. More recently, position errors ${ }^{14-16}$, background ${ }^{17,18}$, noise statistics ${ }^{19,20}$ and partially coherent illumination ${ }^{21-25}$, or vibrations have been added to the nonlinear optimization to fit the data.

Existing methods iterate between an object space - an image representing an estimate of the object - and a measurement space given by the measured diffraction frames. For example in the approach first described by ${ }^{14}$, one starts from an estimate of the positions of the illuminating beam and an estimate of the object under study. From this starting point one minimizes the discrepancy with the data using local - gradient based - optimization and obtains a new estimate of the object and positions.

In this paper we introduce an additional optimization step in the measurement space which is -since in ptychographic experiments one records multiple views of the same region of the object - of higher dimensionality than the object space. We form pairwise comparisons between neighboring frames and update the unknown parameters of each diffraction frame so that each frame is consistent with each other.

The approach described in this work achieves accelerated convergence for large scale phase retrieval problems spanning multiple length-scales. We also show that this approach can recover experimental fluctuations over a large range of time-scales. 

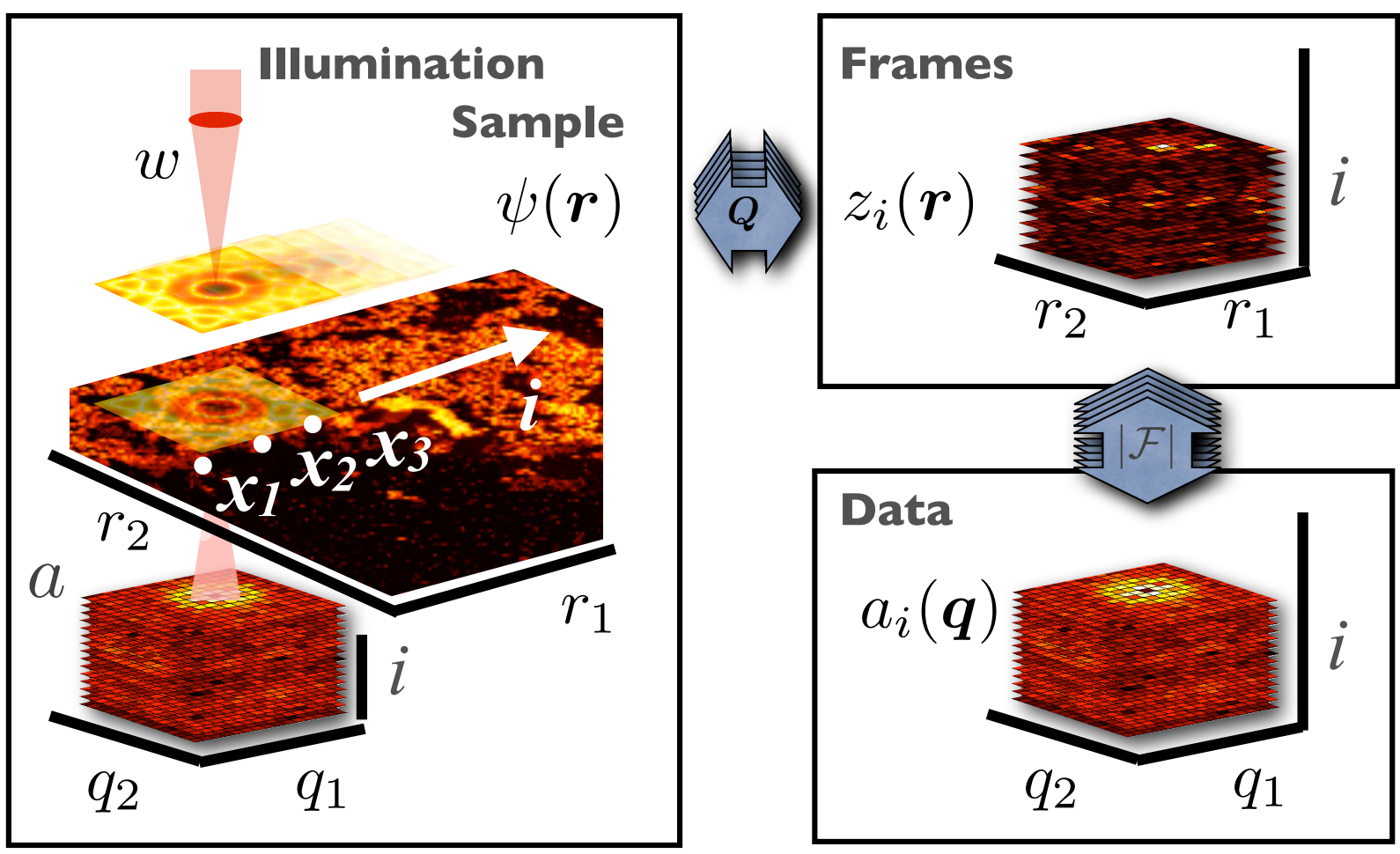

FIG. 1: (Left) Experimental geometry in ptychography: An unknown sample with transmission $\psi(\boldsymbol{r})$ is rastered through an illuminating beam $w(\boldsymbol{r})$, and a sequence of diffraction measurements $\left|a_{(i)}\right|^{2}$ are recorded on an area detector as the sample is rastered around. The point-wise product between illuminating function and sample $z_{(i)}(\boldsymbol{r})=w(\boldsymbol{r}) \psi\left(\boldsymbol{r}+\boldsymbol{x}_{(i)}\right)$-which we refer to as "frame" throuout the paper- is related to the measurement by a Fourier magnitude relationship $a_{(i)}=\left|\mathcal{F} z_{(i)}\right|$.

\section{Notation and background}

In a ptychography experiment ${ }^{3,7,8,10,26-28}$ (see Fig. 1), a two dimensional small beam with distribution $w(\boldsymbol{r})$ of dimension $m \times m$ illuminates an subregion centered at $\boldsymbol{x}_{(i)}$-referred to as frame- of an unknown object of interest $\psi$ of dimension $n \times n$. Here $0<m<n, i=1, \ldots, k$, $k$ is the number of frames, $r>0$ is a lengthscale (the diffraction limited resolution of the microscope), and

$$
\begin{aligned}
& \boldsymbol{r}=(r \mu, r \nu), \mu, \nu \in\{0, \ldots, m-1\} \\
& \boldsymbol{x}_{(i)}=\left(r \mu^{\prime}, r \nu^{\prime}\right), \mu^{\prime}, \nu^{\prime} \in\{0, \ldots, n-m\} .
\end{aligned}
$$

As $\boldsymbol{x}_{(i)}$ is rastered on a typically coarser grid, $\boldsymbol{r}+\boldsymbol{x}_{(i)}$ spans a finer grid of dimension $n \times n$. Here for simplicity we consider square matrices, and generalization to non-square matrices is straightforward but requires more indices and complicates notation. In other words, we assume that a sequence of $k$ diffraction intensity patterns $\mathcal{I}_{(i)}(\boldsymbol{q})=a_{(i)}^{2}(\boldsymbol{q})$ are collected as the position of the object is rastered on the position $\boldsymbol{x}_{(i)}$, where

$$
\boldsymbol{q}=\left(\frac{2 \pi}{m r} \mu, \frac{2 \pi}{m r} \nu\right), \mu, \nu \in\{0, \ldots, m-1\} .
$$

The relationship among the amplitude $a_{(i)}$, the probe $w$ and an unknown object $\psi$ to be estimated can be expressed as follows:

$$
\begin{aligned}
& a_{(i)}(\boldsymbol{q})=\left|\mathcal{F} w(\boldsymbol{r}) \psi\left(\boldsymbol{r}+\boldsymbol{x}_{(i)}\right)\right|, \\
& (\mathcal{F} f)(\boldsymbol{q})=\sum_{\boldsymbol{r}} e^{i \boldsymbol{q} \cdot \boldsymbol{r}} f(\boldsymbol{r}),
\end{aligned}
$$

where the sum over $\boldsymbol{r}$ is given on all the indices $m \times m$ of $\boldsymbol{r}$, and $\mathcal{F}$ is the two dimensional discrete Fourier transform.

We introduce the illumination operator $Q_{(i)}, i=$ $1,2, \cdots, k$, associated with $\boldsymbol{x}_{(i)}$ that extracts a frame $z_{(i)}$ out of $\psi$, and scales the frame point-wise by the illumination function $w(\boldsymbol{r})$ (see Fig. 1):

$$
z_{(i)}(\boldsymbol{r})=w(\boldsymbol{r}) \psi\left(\boldsymbol{r}+\boldsymbol{x}_{(i)}\right)=\left[Q_{(i)} \psi\right](\boldsymbol{r}),
$$

where $z_{(i)}$ represents the frames extracted from $\psi$ and multiplied by the probe $w(\boldsymbol{r})$.

To have a compact representation for numerics, we introduce the following notations. We represent $\psi$ as a vector of length $n^{2}$, that is, $\psi \in \mathbb{C}^{n^{2}}$. The moving beam associated with the illumination function $w(\boldsymbol{r})$ can be represented as an $m^{2} \times n^{2}$ sparse "illumination matrix" associated with the illumination operator, which is again denoted as $Q_{(i)}$. To express $Q_{(i)}$ in the matrix form, we 
introduce a restriction matrix $R_{(i)}$, which restricts the $n \times n$ region onto the $m \times m$ subregion centered at $\boldsymbol{x}_{(i)}$, that is,

$$
Q_{(i)}=\operatorname{diag}(w) R_{(i)} .
$$

The relationship between the diffraction measurements collected in a ptychography experiment and the unknown object to be recovered can be represented compactly as:

$$
\boldsymbol{a}=|\boldsymbol{F} \boldsymbol{Q} \psi|,
$$

if we stack the diffraction measurements $a_{(i)}$ into a long vector $\boldsymbol{a}$, and define various matrices as follows:

$$
\begin{aligned}
& \boldsymbol{a}=\left(\begin{array}{c}
a_{(1)} \\
\vdots \\
a_{(k)}
\end{array}\right), \boldsymbol{Q}=\left(\begin{array}{c}
Q_{(1)} \\
\vdots \\
Q_{(k)}
\end{array}\right), \\
& \boldsymbol{z}=\left(\begin{array}{c}
z_{(1)} \\
\vdots \\
z_{(k)}
\end{array}\right), \boldsymbol{F}=\left(\begin{array}{ccc}
\mathcal{F} & & \\
& \ddots & \\
& & \mathcal{F}
\end{array}\right) .
\end{aligned}
$$

We call the domain of $\boldsymbol{Q}$ the object space, and the range of $\boldsymbol{F} \boldsymbol{Q}$ the measurement space.

Geometrically, $\boldsymbol{Q}$ is the matrix that extracts $k$ frames out of an object $\psi$ and multiplies them by the probe $w ; \boldsymbol{Q}^{*}$ is the conjugate transpose that merges $k$ frames onto the object space; in addition, $\boldsymbol{Q}^{*} \boldsymbol{Q}$ can be viewed as the normalization factor given by the sum of the illumination functions. In particular, by a direct calculation, $\boldsymbol{Q}^{*} \boldsymbol{Q}$ is a $n \times n$ diagonal matrix whose $l$-th diagonal entry is $\sum_{k: l=\boldsymbol{x}_{k}+\boldsymbol{r}_{k}}\left|w\left(\boldsymbol{r}_{k}\right)\right|^{2}$, where we abuse the notation by using $l$ to indicate the point on the object space. Physically $l$ is the index of the grid point on the unknown object of interest which is covered by the point $\boldsymbol{r}_{k}$ of the $k$-th illumination window. See table I for the relationship between probe $w$, translation $\boldsymbol{x}_{(i)}, Q_{(i)}$ and $\boldsymbol{Q}$.

\begin{tabular}{|l|l|}
\hline$Q_{(i)} \psi$ & $\operatorname{diag}(w) R_{(i)} \psi$ \\
\hline$Q_{(i)}^{*} z_{(i)}$ & $R_{(i)}^{*} \operatorname{diag}(\operatorname{conj}(w)) z_{(i)}$ \\
\hline $\boldsymbol{Q}^{*} \boldsymbol{z}$ & $\sum_{i} R_{(i)}^{*} \operatorname{diag}(\operatorname{conj}(w)) z_{(i)}$ \\
\hline$e_{l}^{T} \boldsymbol{Q}^{*} \boldsymbol{Q} e_{k}$ & $\sum_{\boldsymbol{x}_{k}+\boldsymbol{r}_{k}=l}\left|w\left(\boldsymbol{r}_{k}\right)\right|^{2} \delta_{l, k}$ \\
\hline$e_{l}^{T}\left(\boldsymbol{Q}^{*} \boldsymbol{Q}\right)^{-1} \boldsymbol{Q}^{*} \boldsymbol{z}$ & $\frac{e_{l}^{T} \sum_{i} R_{(i)}^{*} \operatorname{diag}\left(\operatorname{conj}(w) z_{(i)}\right.}{\sum_{\boldsymbol{x}_{k}+\boldsymbol{r}_{k}=l}\left|w\left(\boldsymbol{r}_{)}\right)\right|^{2}}$ \\
\hline$P_{\boldsymbol{F}}^{\boldsymbol{a}_{(i)}} z_{(i)}$ & $\sum_{\boldsymbol{q}} e^{-i \boldsymbol{q} \cdot \boldsymbol{r} \cdot} \frac{\sum_{\boldsymbol{r}} e^{i \boldsymbol{q} \cdot \boldsymbol{r}} z_{(i)}(\boldsymbol{r})}{\left|\sum_{\boldsymbol{r}} e^{i \boldsymbol{q} \cdot \boldsymbol{r}} z_{(i)}(\boldsymbol{r})\right|} \boldsymbol{a}_{(i)}$ \\
\hline
\end{tabular}

TABLE I: Linear algebra notation. Here $e_{l}$ is the unit $n \times 1$ vector with the $l$-th entry 1 and $\delta$ is the Kronecker delta. The division is understood as an element-wise operation. The operator $P_{\boldsymbol{F}}^{\boldsymbol{a}_{(i)}}$ is defined in (8)

The objective of the ptychographic reconstruction problem is to find $\psi$ given $\boldsymbol{a}$ from (Eq. 1). This is often formulated using a "divide and conquer" approach referred to as projection algorithms, iterative transform methods, or alternating direction methods ${ }^{29}$. One formulates the relationship (Eq. 1) as:

$$
\begin{aligned}
& \boldsymbol{a}=|\boldsymbol{F} \boldsymbol{z}|, \\
& \boldsymbol{z}=\boldsymbol{Q} \psi .
\end{aligned}
$$

These algorithms are often defined in terms of two projection operators $P_{F}^{a}$ and $P_{Q}$ that project onto the solution $z$ to Eqs. (2 and 3) that is closest to the current estimate described in Section II.

Alternative approaches include formulating the problem as:

$$
\min _{\psi}\|\boldsymbol{a}-|\boldsymbol{F} \boldsymbol{Q} \psi|\|
$$

and solve it by standard unconstrained minimization algorithms such as conjugate gradient, Newton and quasiNewton methods ${ }^{14,19,30}$. Efficient projection operators can be used if we formulate Eq. 4 using the frames $\boldsymbol{z}$ as slack variables as discussed in Section II and solve:

$$
\min _{\boldsymbol{z}}\|\boldsymbol{a}-|\boldsymbol{F} \boldsymbol{z}|\|
$$

with the conditions that $\boldsymbol{z}$ satisfies Eq. (3) using projected gradient, Newton and quasi-Newton methods ${ }^{30,31}$.

Another approach uses a $n^{2} \times n^{2}$ phase-space described in terms of a "Wigner Distribution" function ${ }^{3,7}$. More recently ${ }^{32-34}$ a convex relaxation of the quadratic problem is obtained by lifting to an $n^{2} \times n^{2}$ space and minimizing the rank of the matrix

\section{Main results}

The main contribution of this paper is the introduction of an additional optimization step in the measurement space, which with a dimensionality of $\mathrm{km}^{2}$, is larger than the object space, $n^{2}$. It is aimed to deal with fluctuating intensities, position errors, poor calibration using multiplexing illumination, and an unknown offset (background) for every pixel but constant throughout the acquisition (or vice versa). Specifically, instead of solving Eq. (4), we wish to minimize the gap between the measurement space and the smaller object space:

$$
\min _{\boldsymbol{z},|\boldsymbol{F} \boldsymbol{z}|=\boldsymbol{a}}\left\|\left[I-P_{\boldsymbol{Q}}\right] \boldsymbol{z}\right\|,
$$

where $I$ is the identity operator and $P_{Q}$ represents a projection onto the object space and will be described in the following section.

Recently it was proposed to use maxcut algorithms to solve a similar problem ${ }^{35}$. Here we approach the problem differently. We start from the redundant measurement space and compute pairwise comparison between frames before merging into the object space.

In section III we consider the case that the diffraction measurement $a_{(i)}$ is contaminated (i.e. multiplied) by a unknown scalar factor $\omega_{(i)}$. We encounter this problem if the intensity or integration time of the incident beam 
is unknown. If we fix the relative amplitude and phase within every frame $z_{(i)}$ and minimize the gap Eq. (6) with respect to the vector $\boldsymbol{\omega}$ for a given $\boldsymbol{z}$, we can express Eq. (6) as:

$$
\begin{aligned}
& \min _{\boldsymbol{\omega}} \boldsymbol{\omega}^{*} H \boldsymbol{\omega} \\
& H_{i, j}=z_{(i)}^{*}\left(\delta_{i, j} I-\frac{Q_{(i)} Q_{(j)}^{*}}{e_{i}^{T}\left(\boldsymbol{Q}^{*} \boldsymbol{Q}\right) e_{j}}\right) z_{(j)},
\end{aligned}
$$

where the $k \times k$ matrix $H$ is calculated by computing the pairwise dot product between overlapping frames. While this problem arises from the need to account for intensity fluctuations, it turns out to be a useful technique to improve the convergence rate for large scale problems. The phase vector obtained by normalizing $\boldsymbol{\omega}$ enables us to adjust for the relative phase between frames and accelerate the rate of convergence in iterative algorithms.

We use a similar approach to optimize perturbations of the illumination matrix $\boldsymbol{Q}$ : the position among frames (Section IV). Alternative approaches optimizing the positions from the reduced object space have been proposed by others ${ }^{14,15}$. By minimizing the gap between measurement space and constraint, we obtain a first order correction formula that relies on pairwise scalar products between neighboring frames. We expect a method based on pairwise comparsions to work well in large scale problems when long range position drifts may arise.

In Section VI we report the following numerical results:

- Exact reconstruction with intensity fluctuation given by the coefficients $\omega_{(i)}$ (see Fig. 4).

- Accelerated convergence (Fig. 5) even when no intensity fluctuation is present in the data (Table III)

- Exact reconstruction with multiplexing using 4 simultaneous illuminations adding incoherently on the detector, with perturbation of the amplitudes (Fig. 6)

- Position recovery (Figs. 7) of the illuminating probe.

- Joint reconstruction of the sample and fluctuating background noise independent from the sample (Figs. 8,9).

- Exact reconstruction with missing (corrupted) data entries (see Fig. 11,12).

In the following section II we will describe the standard operators commonly used in the literature.

\section{STANDARD PROJECTION ALGORITHMS}

The projection operator $P_{F}^{a}$ mentioned in the previous section is often known as the Fourier magnitude projection operator. Applying this operator to a vector $\boldsymbol{z}$ yields

$$
P_{F}^{a} \boldsymbol{z}=\boldsymbol{F}^{*}\left(\frac{\boldsymbol{F} \boldsymbol{z}}{|\boldsymbol{F} \boldsymbol{z}|} \cdot \boldsymbol{a}\right) .
$$

where division and multiplication are intended as element-wise operations. It is easy to verify that $\left|P_{F}^{a} \boldsymbol{z}\right|=$ $|\boldsymbol{a}|$ and therefore $P_{F}^{\boldsymbol{a}} \boldsymbol{z}$ satisfies Eq. (2) for any $\boldsymbol{z}$. We mention that $P_{F}^{a}$ is a projection in the sense that

$$
\begin{array}{r}
P_{F}^{a} \boldsymbol{z}=\arg \min _{\bar{z}}\left\|\boldsymbol{z}_{(i)}-\overline{\boldsymbol{z}}_{(i)}\right\|, \\
\text { subject to }|\boldsymbol{F} \overline{\boldsymbol{z}}|=\boldsymbol{a},
\end{array}
$$

where \|\| denotes the Euclidean norm. The matrix $\boldsymbol{Q}$ defines an orthogonal projection operator $P_{\boldsymbol{Q}}$ that projects any vector in $\mathbb{C}^{k m^{2}}$ onto the range of $\boldsymbol{Q}$ :

$$
P_{Q}=\boldsymbol{Q}\left(\boldsymbol{Q}^{*} \boldsymbol{Q}\right)^{-1} \boldsymbol{Q}^{*},
$$

when $\left(\boldsymbol{Q}^{*} \boldsymbol{Q}\right)^{-1}$ exists. An alternative formulation uses projection operators that apply on the Fourier frames $\hat{z}=\boldsymbol{F} \boldsymbol{z}$ :

$$
\tilde{P}_{\boldsymbol{Q}}=\boldsymbol{F} P_{\boldsymbol{Q}} \boldsymbol{F}^{*}, \quad \tilde{P}_{F}^{a}=\boldsymbol{F} P_{F}^{a} \boldsymbol{F}^{*} .
$$

Line search stratrategies to solve Eq. (9) can be implemented more efficiently using this formulation ${ }^{30}$.

In the simple alternating projection algorithm, the approximation to the solutions of (Eqs. (2) and (3)) are updated by:

$$
\boldsymbol{z}^{(\ell)}=\left[P_{\boldsymbol{Q}} P_{F}^{\boldsymbol{a}}\right] \boldsymbol{z}^{(\ell-1)},
$$

where typically the initial guess $\boldsymbol{z}^{(0)}$ is a random vector. Clearly $P_{F}^{\boldsymbol{a}}$ from (9) forces $\boldsymbol{z}^{(\ell-1)}$ to have the right amplitude in the Fourier domain, and $P_{\boldsymbol{Q}}$ forces $P_{F}^{a} \boldsymbol{z}^{(\ell-1)}$ to be located in the range of $\boldsymbol{Q}$. We note that the projector $P_{\boldsymbol{Q}}$ can be expressed by computing the running estimate of $\psi$ denoted as $\psi^{(\ell)}$

$$
\psi^{(\ell)}=\arg \min _{\psi}\left\|P_{F}^{a} \boldsymbol{z}^{(\ell-1)}-\boldsymbol{Q} \psi\right\|,
$$

which is solved by taking Eq. (10) into account:

$$
\psi^{(\ell)}=\left[\left(\boldsymbol{Q}^{*} \boldsymbol{Q}\right)^{-1} \boldsymbol{Q}^{*}\right] P_{F}^{a} \boldsymbol{z}^{(\ell-1)},
$$

when $\left(\boldsymbol{Q}^{*} \boldsymbol{Q}\right)^{-1}$ exists. Notice that $P_{\boldsymbol{Q}} P_{F}^{\boldsymbol{a}} \boldsymbol{z}^{(\ell-1)}=\boldsymbol{Q} \psi^{(\ell)}$.

We mention two practical issues regarding the updating steps (12) and (9). First, since $\left(\boldsymbol{Q}^{*} \boldsymbol{Q}\right)^{-1}$ may not exist, one may introduce a regularization factor $\epsilon$ into (12) and update the running estimate as:

$$
\psi^{(\ell)}=\left(\boldsymbol{Q}^{*} \boldsymbol{Q}+\epsilon\right)^{-1}\left(\boldsymbol{Q}^{*} P_{F}^{a} \boldsymbol{z}^{(\ell-1)}+\epsilon \psi^{(\ell-1)}\right) .
$$

with typically $\psi^{(0)}=0 . \epsilon$ is a regularization factor that leaves unchanged the entries that are never illuminated, and gradually reduces the correction from $\psi^{(\ell-1)}$ where the sum of the illuminating probe intensities is small. If we replace Eq. (13) by Eq. (14) we obtain an operator that is no longer a projection operator but can be viewed as a relaxed projection.

If the entries of $a_{(i)}(\boldsymbol{q})$ are corrupted by gaussian random noise with known variance $\boldsymbol{\sigma}_{(i)}^{2}(\boldsymbol{q})$, which is expressed as a $m^{2}$ long column vector, one may replace Eq. (9) modifying $P_{F}^{a}$ with 


$$
P_{F}^{(\boldsymbol{a}, \sigma)} \boldsymbol{z}=\boldsymbol{F}^{*}\left(\frac{\boldsymbol{F} \boldsymbol{z}}{|\boldsymbol{F} \boldsymbol{z}|} \cdot \frac{\boldsymbol{a}+|\boldsymbol{F} \boldsymbol{z}| \boldsymbol{\sigma}^{2}}{1+\boldsymbol{\sigma}^{2}}\right),
$$

where $\boldsymbol{\sigma}$ is viewed as a regularization factor that leaves unchanged the entries of $\boldsymbol{z}$ when the data entries are completely corrupted $\left(\boldsymbol{\sigma}^{2} \rightarrow \infty\right)$. Clearly $\frac{\boldsymbol{a}+|\boldsymbol{F} \boldsymbol{z}| \boldsymbol{\sigma}^{2}}{1+\boldsymbol{\sigma}^{2}} \frac{1}{|\boldsymbol{F} \boldsymbol{z}|} \rightarrow 1$ when $\boldsymbol{\sigma}^{2} \rightarrow \infty$. When $\boldsymbol{\sigma}^{2} \rightarrow 0$, (Eq 15$)$ reverts to the regular projection operator (Eq. 8).

The simple alternating projection algorithm can be viewed as the projected steepest descent algorithms. Projected conjugate gradient methods have also been proposed in ${ }^{14,19,30}$ to accelerate convergence rate.

A number of heuristic first order algorithms have been proposed that outperform the simple algorithms, a few examples are given in Tab. II, with $\beta \in[0,1]$ is a relaxation parameter. Very recently, an alternating direction method (ADM) was proposed to work with a special augmented Lagrangian function ${ }^{29}$. This function is minimized by applying a block coordinate descent scheme ${ }^{36}$ (or alternating search directions) akin to these projection operators.

\section{FLUCTUATING INTENSITIES, AND AUGMENTED PHASE RETRIEVAL}

Intensity fluctuations can be accounted for by introducing a scalar scaling factor $\omega_{i} \in \mathbb{C}$ multiplying every $\left(\mathrm{m}^{2}\right)$ pixel of a diffraction frame. This can be expressed in various forms (frame by frame or all at once) as:

$$
\begin{aligned}
\left|\left(\mathcal{F} z_{(i)}\right)\right| \cdot\left|\omega_{(i)}\right| & =a_{(i)}, \quad \forall i \\
|\operatorname{diag}(\boldsymbol{F} \boldsymbol{z}) \boldsymbol{B} \boldsymbol{\omega}| & =\boldsymbol{a}, \quad(\boldsymbol{B})_{i, j}=\delta_{i, j} 1_{m^{2}},
\end{aligned}
$$

where $\boldsymbol{\omega}=\left[\omega_{1}, \ldots, \omega_{k}\right] \in \mathbb{C}^{k}$ and $\boldsymbol{B}$ is a $k \times k$ diagonal block matrix with the diagonal entry $1_{m^{2}}$, which is the $m^{2} \times 1$ matrix with 1 in all entries. In other words, $\boldsymbol{B}$ copies the scalar factors $\omega_{i}$ before multiplying by $\boldsymbol{F} \boldsymbol{z}$. In practice $\omega_{i}$ is unknown and needs to be estimated. If we know $\boldsymbol{z}$ or its approximation, to estimate $\omega_{i}$, we find the vector $\boldsymbol{\omega}$ that minimizes the gap with the object space:

$$
\arg \min _{\boldsymbol{\omega}}\left\|\left(I-P_{\boldsymbol{Q}}\right) \operatorname{diag}(\boldsymbol{z}) \boldsymbol{B} \omega\right\|^{2} .
$$

We can write Eq. (18) as:

$$
\begin{aligned}
& \arg \min _{\boldsymbol{\omega}} \boldsymbol{\omega}^{*} H \boldsymbol{\omega}, \\
H_{i, j}= & \mathbf{1}^{T} \operatorname{diag}\left(z_{(i)}^{*}\right)\left(\delta_{i, j} I-\frac{Q_{(i)} Q_{(j)}^{*}}{e_{i}^{T}\left(\boldsymbol{Q}^{*} \boldsymbol{Q}\right) e_{j}}\right) \operatorname{diag}\left(z_{(j)}\right) \mathbf{1}, \\
= & z_{(i)}^{*}\left(\delta_{i, j} I-\frac{Q_{(i)} Q_{(j)}^{*}}{e_{i}^{T}\left(\boldsymbol{Q}^{*} \boldsymbol{Q}\right) e_{j}}\right) z_{(j)},
\end{aligned}
$$

where the $k \times k$ matrix $H$ is computed by performing the scalar product between every pair of overlapping frames. We can eliminate the trivial solution $\boldsymbol{\omega}=0$ by setting an additional constraint such as $\sum \boldsymbol{\omega}$, or $\|\boldsymbol{\omega}\|=$ constant. A simple way to solve this problem (see appendix A) is to start with $\mathbf{1}$ as our first guess for $\omega$ and solve:

$$
H \boldsymbol{\omega}=\alpha \mathbf{1},
$$

where $\alpha$ is chosen to normalize the average flux $\|\boldsymbol{\omega}\| /\|\mathbf{1}\|$.

In order to take the intensity fluctuation problem into account while applying the projection algorithm (2) and (3), we introduce the following solution. First, we replace the operator $P_{Q}$ used in the standard projection algorithms listed in Table II by an augmented projection operator $P_{\boldsymbol{Q}}^{\boldsymbol{\omega}}$ defined as:

$$
P_{\boldsymbol{Q}}^{\boldsymbol{\omega}}=D_{\boldsymbol{\omega}}^{-1} P_{\boldsymbol{Q}} D_{\boldsymbol{\omega}}, \quad D_{\boldsymbol{\omega}}=\operatorname{diag}(\boldsymbol{\omega}),
$$

where $\boldsymbol{\omega}$ is the solution to Eq.(19) or Eq.(20). An alternative modification is to recompute the normalization factor $\boldsymbol{Q}^{*} \boldsymbol{Q}$ with the scaling factors $\left|\omega_{i}\right|^{2}$. This yields an orthogonal projection:

$$
\bar{P}_{\boldsymbol{Q}}^{\omega}=\boldsymbol{Q}_{\boldsymbol{\omega}}\left(\boldsymbol{Q}_{\omega}^{*} \boldsymbol{Q}_{\boldsymbol{\omega}}\right)^{-1} \boldsymbol{Q}_{\boldsymbol{\omega}}^{*}, \quad \boldsymbol{Q}_{\boldsymbol{\omega}}=D_{\omega}^{-1} \boldsymbol{Q}
$$

When no intensity fluctuations are present, we normalize $\boldsymbol{\omega}$ and replace $D_{\boldsymbol{\omega}}$ with:

$$
D_{\hat{\omega}}=\operatorname{diag}\left(\frac{\omega}{|\boldsymbol{\omega}|}\right) .
$$

Although the construction of $P_{\boldsymbol{Q}}^{\boldsymbol{\omega}}$ is motivated by the need to account for intensity fluctuations among different diffraction frames in the measured data, it turns out to be a useful technique for accelerating the convergence of projection algorithms even when no intensity fluctuation is present in the data. The minimization problem in (Eq. 18) is similar to the phase problem of how to merge frames $\boldsymbol{z}_{(i)}$ with unknown phase factor $\boldsymbol{\omega}$, which can be written as:

$$
\arg \min _{\boldsymbol{\omega},\left|\omega_{i}\right|=1} \boldsymbol{\omega} H \boldsymbol{\omega}
$$

Replacing the condition $|\boldsymbol{\omega}|=\mathbf{1}$ with weaker conditions such as $\sum \boldsymbol{\omega}$ or $\|\boldsymbol{\omega}\|=$ constant enables us to solve this problem more efficiently. A similar approach is discussed in 39 .

The problem of the incoherent superposition of different signals can be treated in a similar way.

\section{A. Multiplexing and incoherent measurements}

The incoherent measurement model is as follows. We consider $z_{i}$ the highly redundant set of frames generated for all the positions of the illumination function during an exposure. For example, a single exposure $a_{(i)}$ may represents the sum of the intensities generated by an illumination beam that translates during the exposure, or may represent a binned sample of a continuous signal. Assume we have $k$ redundant measurement $\boldsymbol{z}_{(i)}$, where $i=1, \ldots, k$. The incoherent measurement is introduced 


\begin{tabular}{|l|l|}
\hline projection algorithm & updating formula $\boldsymbol{z}^{(\ell+1)}=$ \\
\hline \hline Alternating Projection $^{37}$ & {$\left[P_{Q} P_{F}\right] \boldsymbol{z}^{(\ell)}$} \\
\hline HIO $^{37}$ & {$\left[P_{Q} P_{F}+\left(I-P_{Q}\right)\left(I-\beta P_{F}\right)\right] \boldsymbol{z}^{(\ell)}$} \\
\hline Difference Map $^{8}$ & {$\left[P_{F} P_{Q}+\left(I-P_{F}\right)\left(I-\beta P_{Q}\right)\right] \boldsymbol{z}^{(\ell)}$} \\
\hline RAAR $^{38}$ & {$\left[2 \beta P_{Q} P_{F}+(1-2 \beta) P_{F}+\beta\left(P_{Q}-I\right)\right] \boldsymbol{z}^{(\ell)}$} \\
\hline
\end{tabular}

TABLE II: Popular fix-point algorithms used in phase retrieval. HIO: hybrid input-output algorithm. RAAR: Relaxed averaged alternating reflections algorithm.

by summing $s<k$ illumination windows according to a weight factor, where we assume $\mathrm{k} / \mathrm{s}$ is an integer for convenience. The weight factor, or the integration time, for each frame, is represented by $|\boldsymbol{\omega}|^{2}$. In particular, the redundant set of frames $z_{(i)}$ is not measured directly; instead it is multiplied by a known averaging operator, which is expressed as $|\boldsymbol{\omega}|^{2}$ and a $(k / s) m^{2} \times k m^{2}$ real matrix $\boldsymbol{\Omega}$ with all non-zero entries 1 . Geometrically, $\boldsymbol{\Omega}$ groups the frames, which are weighted by $|\boldsymbol{\omega}|^{2}$, and then the weighted frames in each group are summed. The incoherent measurement can thus be expressed by modifying (17):

$$
\boldsymbol{a}^{2}=\boldsymbol{\Omega}\left(|\boldsymbol{F} \boldsymbol{z}|^{2} \cdot \boldsymbol{B}|\omega|^{2}\right),
$$

where $\cdot$ and $|\cdot|^{2}$ are intended as elementwise operations $\boldsymbol{z}$ is a given $\mathrm{km}^{2}$ complex column vector and $\boldsymbol{a}^{2}$ is a $(k / s) m^{2}$ real column vector. The projection operator associated with this problem can be expressed as follows:

$$
P_{F}^{a, \boldsymbol{\Omega}} \boldsymbol{z}=\boldsymbol{F}^{*}\left(\operatorname{diag}\left(\sqrt{\boldsymbol{\Omega}^{*} \frac{\boldsymbol{a}^{2}}{\boldsymbol{\Omega}\left(|\boldsymbol{F} \boldsymbol{z}|^{2} \cdot \boldsymbol{B}|\omega|^{2}\right)}}\right) \boldsymbol{F} \boldsymbol{z}\right),
$$

when all non-zero entries of $\boldsymbol{\Omega}$ are 1 and $\boldsymbol{\Omega} \boldsymbol{\Omega}^{*}=$ $s \boldsymbol{I}_{(k / s) m^{2}}$. Here $\boldsymbol{\Omega}^{*}$ copies the entries over all the frames that contribute to an exposure $a_{(i)}$. We can directly check that Eq. (25) satisfies Eq. (24). Replacing this operator in the reconstruction process is a subject of recent interest by several groups ${ }^{21,40}$. Other approaches for incoherent averaging over wavelengths, orientation, coherence, etc. have been discussed by others ${ }^{22},{ }^{23},{ }^{24},{ }^{25}$.

If $|\boldsymbol{\omega}|^{2}$ is unknown, we can derive it from solving a minimization problem of the type Eq. (4), from the object space, but with an incoherent measurement model. Another approach is to obtain $\boldsymbol{\omega}$ by solving a minimization problem of the type Eq. (6), from the measurement space with the incoherent measurement model by solving Eq.20 and using Eq. 21 .

Numerical tests described in section VI show the exact recovery (within numerical precision) of the object and a multiplexing array of beam positions averaged incoherently with errors in the calibration of the amplitude factors. The ability to recover the relative amplitude of a redundant set of frames that are averaged during the measurement enables us also to identify which instance of the experimental parameters occurred, or to recover or calibrate the amplitude coefficients of a multiplexing array of incident beams.
The number of frames used in the calculation however increases, and with it, the computational cost increases as well. To reduce the number of parameters to optimize we can describe the change in measurement space using Taylor expansion.

\section{POSITION RETRIEVAL}

We consider the case in which the probe $w$ is translated from the input coordinate by an unknown distance $\xi$. We call $\boldsymbol{Q}_{\xi}$ the unknown illumination matrix used to generate the data. To determine the illumination matrix, we determine the parameter $\xi$ so that the error $\varepsilon_{\boldsymbol{Q}_{\xi}}$ is minimized:

$$
\varepsilon_{\boldsymbol{Q}_{\xi}}:=\left\|\left[I-P_{\boldsymbol{Q}_{\xi}}\right] \boldsymbol{z}\right\|^{2} .
$$

Given the illumination function $w$, we can compute the first and second order derivatives with respect to translation.

We denote by $Q_{(i)}, R_{1,(i)}, R_{2,(i)}, S_{11,(i)}, S_{12,(i)}, S_{21,(i)}$ and $S_{22,(i)}$ the illumination matrices that extract a frame out of an image and multiply by $w_{(i)}(\boldsymbol{r}), \partial_{x_{1}} w_{(i)}(\boldsymbol{r})$, $\partial_{x_{2}} w_{(i)}(\boldsymbol{r}), \partial_{x_{1}}^{2} w_{(i)}(\boldsymbol{r}), \partial_{x_{1}, x_{2}}^{2} w_{(i)}(\boldsymbol{r}), \partial_{x_{2}, x_{1}}^{2} w_{(i)}(\boldsymbol{r}) \quad$ and $\partial_{x_{2}}^{2} w_{(i)}(\boldsymbol{r})$ respectively. Build up $\boldsymbol{Q}, \boldsymbol{R}_{1}, \ldots, \boldsymbol{S}_{22}$ from $Q_{(i)}, R_{1,(i)}, \ldots, S_{22,(i)}$, which are tall and skinny matrices of the same size as $\boldsymbol{Q}$ discussed earlier, with identical location of the non-zero entries. Assume that $\boldsymbol{Q}_{\xi}$ satisfies the following second order perturbation from $\boldsymbol{Q}$ :

$$
\begin{aligned}
& \boldsymbol{Q}_{\xi}=\boldsymbol{Q}+\operatorname{diag}\left(\boldsymbol{B} \boldsymbol{\xi}_{1}\right) \boldsymbol{R}_{1}+\operatorname{diag}\left(\boldsymbol{B} \boldsymbol{\xi}_{2}\right) \boldsymbol{R}_{2} \\
& +\operatorname{Diag}\left(\boldsymbol{B} \boldsymbol{\xi}_{1}^{2}\right) \boldsymbol{S}_{11}+2 \operatorname{diag}\left(\boldsymbol{B}\left(\boldsymbol{\xi}_{1} \cdot \boldsymbol{\xi}_{2}\right)\right) \boldsymbol{S}_{\times}+\operatorname{Diag}\left(\boldsymbol{B} \boldsymbol{\xi}_{2}^{2}\right) \boldsymbol{S}_{22}
\end{aligned}
$$

where $\cdot$ and.$^{2}$ are intended as elementwise operations, $\boldsymbol{\xi}_{1}$ (resp. $\boldsymbol{\xi}_{2}$ ) is a $k \times 1$ matrix so that the $i$-th entry is the translation distance in the $x$-axis (resp. $y$-axis) of the $i$ th illumination window, and $\boldsymbol{S}_{\times} \equiv \frac{1}{2}\left(\boldsymbol{S}_{12}+\boldsymbol{S}_{21}\right)$. Using this Taylor expansion into Eq. (26) and setting $\partial_{\xi_{1}}^{*}\|\cdot\|$ and $\partial_{\xi_{2}}^{*}\|\cdot\|$ to 0 gives (see appendix B for the detailed derivation for the 1-dim case.):

$$
\left(\begin{array}{cc}
H_{1} & H_{\times} \\
H_{\times} & H_{2}
\end{array}\right)\left(\begin{array}{c}
\xi_{1} \\
\xi_{2}
\end{array}\right)=\left(\begin{array}{l}
\underline{\boldsymbol{z}}_{i}^{*} \boldsymbol{z}_{\boldsymbol{R}_{1 i}}+\boldsymbol{z}_{\boldsymbol{R}_{1 i}}^{*} \underline{\boldsymbol{z}}_{i} \\
\underline{\boldsymbol{z}}_{i}^{*} \boldsymbol{z}_{\boldsymbol{R}_{2 i}}+\boldsymbol{z}_{\boldsymbol{R}_{2 i}}^{*} \underline{\boldsymbol{z}}_{i}
\end{array}\right),
$$

using the definition $\boldsymbol{z}_{\boldsymbol{R}_{1}, \ldots, \boldsymbol{S}_{22}} \equiv\left[\boldsymbol{R}_{1}, \ldots, \boldsymbol{S}_{22}\right] \frac{1}{\boldsymbol{Q}^{*} \boldsymbol{Q}} \boldsymbol{Q}^{*} \boldsymbol{z}$, $\underline{\boldsymbol{z}} \equiv\left[I-P_{\boldsymbol{Q}}\right] \boldsymbol{z}$, and where the matrices $H_{1}, H_{2}$ and $H_{\times}$ 
are defined as:

$$
\begin{gathered}
\left(H_{1}\right)_{i j}=\left(\boldsymbol{z}_{\boldsymbol{R}_{1 i}}^{*} \boldsymbol{z}_{\boldsymbol{R}_{1 i}}-2 \underline{\boldsymbol{z}}_{i}^{*} \boldsymbol{z}_{\boldsymbol{S}_{11 i}}\right) \delta_{i j}-\boldsymbol{z}_{i}^{*}\left(O_{11}\right)_{i j} \boldsymbol{z}_{j}+\mathrm{cc}, \\
\left(H_{2}\right)_{i j}=\left(\boldsymbol{z}_{\boldsymbol{R}_{2 i}}^{*} \boldsymbol{z}_{\boldsymbol{R}_{2 i}}-2 \underline{\boldsymbol{z}}_{i}^{*} \boldsymbol{z}_{\boldsymbol{S}_{22 i}}\right) \delta_{i j}-\boldsymbol{z}_{i}^{*}\left(O_{22}\right)_{i j} \boldsymbol{z}_{j}+\mathrm{cc}, \\
\left(H_{\times}\right)_{i j}=\left(\boldsymbol{z}_{\boldsymbol{R}_{1 i}}^{*} \boldsymbol{z}_{\boldsymbol{R}_{2 i}}-2 \underline{\boldsymbol{z}}_{i}^{*} \boldsymbol{z}_{S_{\times_{i}}}\right) \delta_{i j}-\boldsymbol{z}_{i}^{*}\left(O_{\times}\right)_{i j} \boldsymbol{z}_{j}+\mathrm{cc},
\end{gathered}
$$

where cc denotes the complex conjugate term,

$$
\begin{gathered}
\left(O_{11}\right)_{i j} \equiv\left(\boldsymbol{R}_{1}\right)_{i} \frac{1}{\boldsymbol{Q}^{*} \boldsymbol{Q}}\left(\boldsymbol{R}_{1}\right)_{j}^{*} \\
\left(O_{22}\right)_{i j} \equiv\left(\boldsymbol{R}_{2}\right)_{i} \frac{1}{\boldsymbol{Q}^{*} \boldsymbol{Q}}\left(\boldsymbol{R}_{2}\right)_{j}^{*} \\
\left(O_{\times}\right)_{i j} \equiv\left(\boldsymbol{R}_{1}\right)_{i} \frac{1}{\boldsymbol{Q}^{*} \boldsymbol{Q}}\left(\boldsymbol{R}_{2}\right)_{j}^{*}
\end{gathered}
$$

The system of equations (Eq. (27)) can be solved efficiently by sparse linear algebra solvers. The entries of the equation are given by the dot product between frames $\left(\boldsymbol{z}, \underline{\boldsymbol{z}}, \boldsymbol{z}_{R_{1}}, \ldots, \boldsymbol{z}_{S_{22}}\right)$ with partial overlap and scaling factors given by $\boldsymbol{R}_{1,2} \frac{1}{\boldsymbol{Q}^{*} \boldsymbol{Q}} \boldsymbol{R}_{1,2}{ }^{*}$. The terms $\underline{\boldsymbol{z}}^{*} \boldsymbol{z}_{\boldsymbol{S}}$ in $H$ are higher order corrections close to the solution and can be neglected in practice. In Section VI we will show that this method can recover the position perturbations to numerical accuracy when the perturbations are smaller than the probe width.

\section{BACKGROUND NOISE}

For completeness we consider an unknown offset $b(\boldsymbol{q}) \geq$ 0 (background) added to each frame. A similar problem is discussed in ${ }^{17}$ where Thurman and Fienup consider the case of a constant signal bias $b(\boldsymbol{q})=b(0)$. Here we extend this approach to a fluctuating background that is different for every pixel but constant throughout the illumination window. When $b$ is constant, the method described here reverts to ${ }^{17,18}$. We express the relationship between the frames $z_{(i)}$, the data $a_{(i)}$ and the background $b$ as:

$$
\left|\hat{z}_{(i)}\right|^{2}+b=a_{(i)}^{2}, \quad \hat{z}_{(i)}=\mathcal{F} z_{(i)} .
$$

A less trivial variation of the problem is when $b_{(i)}(\boldsymbol{q}) \geq 0$ is different for every frame but the same for every pixel $\boldsymbol{q}$.

At each iteration, we solve the following offset minimization problem with an additional scaling parameter:

$$
\begin{array}{r}
\left.\min _{b, \eta} \sum_{i}|| \hat{z}_{(i)}\right|^{2}-\left.\left|\hat{z}_{(i)}^{(\ell)}\right|^{2}\right|^{2} \\
\text { subject to }\left|\hat{z}_{(i)}^{(\ell)}\right|^{2}=\eta^{(\ell)}\left(a_{(i)}^{2}-b^{(\ell)}\right),
\end{array}
$$

where we set the initial value of $b^{(\ell=0)}=0$, and $\eta \in \mathbb{R}^{m^{2}}$ is a shrinkage parameter that accounts for the fact that $\left|\hat{z}^{(\ell)}\right|^{2}$ is on average smaller than $a^{2}$. This is because $\hat{z}^{(\ell)}$ is obtained from a sequence of linear projections that reduce the overall norm. Since $\hat{z}$ is smaller, the solution to the off-set projection problem (29) is biased towards a larger offset. Introducing the shrinkage parameter $\eta$ equal for every frame provides the flexibility to avoid this problem.
By solving for $\eta$ first, we obtain the first and second order terms:

$$
\eta^{(\ell)}=\frac{\sum_{i} d_{(i)}\left|\hat{z}_{(i)}^{(\ell)}\right|^{2}}{\sum_{i} d_{(i)}^{2}},
$$

where $d_{(i)}=a_{(i)}^{2}-b$. Solving for $b$ for a fixed $\eta$ gives

$$
\begin{aligned}
b^{(\ell)}-b^{(\ell-1)} & =\frac{1}{k} \sum_{i}\left(d_{(i)}-\left|\hat{z}_{(i)}^{(\ell)}\right|^{2} \frac{1}{\eta^{(\ell)}}\right) \\
& =\left\langle d_{(i)}\right\rangle-\frac{1}{\eta^{(\ell)}}\left\langle\left|\hat{z}_{(i)}^{(\ell)}\right|^{2}\right\rangle \\
& =\left\langle d_{(i)}\right\rangle-\frac{\left\langle d_{i}^{2}\right\rangle\left\langle\left|\hat{z}_{(i)}^{(\ell)}\right|^{2}\right\rangle}{\left\langle d_{i}\left|\hat{z}_{(i)}^{(\ell)}\right|^{2}\right\rangle} .
\end{aligned}
$$

To avoid strong perturbations, however, we set $\eta(\boldsymbol{q})=.8$ if $\eta(\boldsymbol{q})<0.8$. When optimizing for a fluctuating offset $\left(b_{(i)}(\boldsymbol{q})=b_{(i)}(0)\right.$ constant for every frame), we simply replace the sum over $i$ with the sum over $\boldsymbol{q}$. The update of $z$ is then computed as a regular Fourier magnitude projection operator with an intensity offset:

$$
\tilde{P}_{F}^{\left(a_{(i)}^{2}-b^{(\ell)}\right)} \hat{z}_{(i)}^{(\ell)}=\hat{z}_{(i)}^{(\ell)} \sqrt{\frac{a_{(i)}^{2}-b^{(\ell)}}{\left|\hat{z}_{(i)}^{(\ell)}\right|^{2}}}
$$

where we used the notation $\tilde{P}=\mathcal{F} P \mathcal{F}^{*}$.

In the following section we will show that common projection methods can recover the background even if the SNR is much smaller than 1.

\section{NUMERICAL TESTS}

The object used to simulate the diffraction pattern is obtained from an SEM image of a cluster of commercial $50 \mathrm{~nm}$ colloidal gold spheres. The image is shown in Fig. 2. The gray scale values were converted to a sample thickness varying between 0 and $50 \mathrm{~nm}$, and we assigned the complex index of refraction of a $750 \mathrm{eV} x$ ray photon going through an organic compound (PMMA). Here the numerical tests are done assuming periodic boundary conditions. These boundary conditions ensure that every region of the object $\psi$ is illuminated with an equal number of overlapping frames, in other words the null space of $Q$ is empty. We use frame width $16 \times 16$, probe width 8 , step size 5 , number of frames $8 \times 8 \ldots 64 \times 64$, RAAR algorithm, $\beta=.75$. The initial guess of the phase chosen to be random. There is no padding of the illumination function shown in Fig. 3 (the intensity measurement is slightly under-sampled).

The metrics $\varepsilon_{F}, \varepsilon_{q}$ used to monitor progress are functions depending on $\boldsymbol{z}^{(\ell)}$ :

$$
\begin{aligned}
& \varepsilon_{F}\left(\boldsymbol{z}^{(\ell)}\right)=\frac{\left\|\left[P_{F}-I\right] \boldsymbol{z}^{(\ell)}\right\|}{\|\boldsymbol{a}\|}, \\
& \varepsilon_{Q}\left(\boldsymbol{z}^{(\ell)}\right)=\frac{\left\|\left[P_{Q}-I\right] \boldsymbol{z}^{(\ell)}\right\|}{\|\boldsymbol{a}\|}
\end{aligned}
$$


where $I$ is the identity operator. This has to be compared to $\varepsilon_{0}$, the error w.r.t the known solution:

$$
\varepsilon_{0}\left(\boldsymbol{z}^{(\ell)}\right)=\frac{1}{\|\boldsymbol{a}\|} \min _{\varphi}\left\|e^{i \varphi} \boldsymbol{z}^{(\ell)}-\boldsymbol{Q} \psi\right\|,
$$

where $\varphi$ is an arbitrary global phase factor.

We report the following observations

- Fluctuating intensities: (Fig. 4) The intensity fluctuation in this tests are 20\%. By solving the new LSQ problem introduced in (21), we obtain accelerated convergence and exact reconstructions every time we tested the problem, see (Fig. 4). No degradation (above numerical precision) introduced by intensities perturbed by $20 \%$.

- Scaling: (Fig. 5) We show improved convergence in the larger scale problems. The results are summarized in Table III. As we increase the number of frames, convergence slows down for standard projection operators. The parameters used in this simulation are $m=16, D_{x}=4, k$ varies and $n=k D_{x}+m$, where $D_{x}$ is the step size of the illumination windows.

- Incoherent Multiplexing: (Fig. 5) Deconvolution of the incoherent sum of frames translated by 3 times the illuminating beam width.

- Incoherent beams with fluctuations: (Fig. 6). Deconvolution of the incoherent sum of frames translated by 3 times the illuminating beam width, with unknown amplitude.

- Positions: (Fig. 7) Recovery of the positions perturbed by an unknown factor randomly distributed between \pm 2.5 pixels.

- Background: (Fig. 8, Fig. 9) $\left\langle\left\|z_{(i)}\right\|\right\rangle_{i} /\|b\|=$ 0.5. In Figure 8,9 we obtain exact reconstruction of the object and background (Background ratio $\|a\| /\|b\|=10^{-6}$ ). Exact recovery (within numerical precision) was obtained with step size $\delta x=3 r$. No degradation (above numerical precision) introduced by the background, nearly no influence on convergence rate.

- Missing data: (Fig. 11, Fig. 12) Exact recovery (within numerical precision) using Eq (15). Frame size:32x32, number of frames: 16x16, step size:3.5 pixels.

\section{CONCLUSIONS}

While phase retrieval problems are notoriously difficult to solve numerically, the high redundancy in ptychographic data enables not only robust phase recovery ${ }^{19,20}$ but the recovery of other parameters such as the

\begin{tabular}{|c|r|r|l|}
\hline \# frames & clock time(s) & iteration & $\varepsilon_{0}^{2}$ \\
\hline standard & & & \\
\hline $4 \times 4$ & 0.7 & 121 & $<1 \mathrm{e}-11$ \\
$8 \times 8$ & 1.4 & 125 & $<1 \mathrm{e}-11$ \\
$16 \times 16$ & 4.9 & 144 & $<1 \mathrm{e}-11$ \\
$24 \times 24$ & 26.3 & 400 & $4.3 \mathrm{e}-10$ \\
$32 \times 32$ & 36.3 & 400 & $4.3 \mathrm{e}-4$ \\
$48 \times 48$ & 90.7 & 400 & $3.4 \mathrm{e}-4$ \\
$64 \times 64$ & 137.5 & 400 & $5.3 \mathrm{e}-3$ \\
\hline augmented & & & \\
\hline $4 \times 4$ & 1.9 & 138 & $<1 \mathrm{e}-11$ \\
$8 \times 8$ & 2.7 & 141 & $<1 \mathrm{e}-11$ \\
$16 \times 16$ & 6.5 & 138 & $<1 \mathrm{e}-11$ \\
$24 \times 24$ & 14 & 134 & $<1 \mathrm{e}-11$ \\
$32 \times 32$ & 25.6 & 139 & $<1 \mathrm{e}-11$ \\
$48 \times 48$ & 60.4 & 142 & $<1 \mathrm{e}-11$ \\
$64 \times 64$ & 96.2 & 149 & $<1 \mathrm{e}-11$ \\
\hline
\end{tabular}

TABLE III: Performance of projection algorithms using matlab R2012a 64-bit (maci64) (lapack version 3.3.1, MKL 10.3.5) on $2 \times 2.2 \mathrm{GHz}$ Quad-core Intel xeon using frames of dimension $16 \times 16$.

illuminating function itself $^{9}$, position ${ }^{14-16}$, coherence function $^{21}$, etc.

In this paper we introduce a modified projection operator for the ptychographic reconstruction problem that accounts for fluctuating intensities, position errors, partial coherence or poor calibration using multiplexing illumination, and an unknown offset (background) difference for every pixel but constant throughout the acquisition (or vice versa). Our approach starts from the redundant measurement space and computes pairwise comparison between frames before merging into the object space. We describe first order methods to minimize the gap between measurement space and object space w.r.t. the perturbation parameter. We show that our method enhances the convergence rate of common projection algorithms. We show several cases where missing information (phases, bad pixels, positions, incoherence, etc.) was retrieved exactly (to within numerical precision) starting from random phases. This method appears to be robust when the amount of overlap between neighboring frames is around $50 \%$ or more.

Further theoretical analysis on the relative merits between object-space minimization and measurement space minimization will be the subject of the future work.

Here some numerical details deserve further developments. By optimizing the phase of each frame from the redundant measurement space, we solve the phase problem at a resolution given by the step size between frames. This intra-frame phase optimization may be applied to merge subregions reconstructed independently by distributed computer systems. For three dimensional objects, we could apply similar approach to merge two 
dimensional views reconstructed independently into one three dimensional object. Finally, this intra-frame optimization could be applied to multi-scale reconstructions where frames are divided in regions of Fourier space, or it could be applied to correct low order phase aberrations between frames. More work is needed to to establish the optimal frequency of communication and the amount of overlap between sub-reconstruction regions.

\section{ACKNOWLEDGMENTS}

The authors thank Prof. Bin Yu and Jeff Donatelli for discussions. This research was supported in part by the Applied Mathematical Sciences subprogram of the Office of Energy Research, U.S. Department of Energy, under contract DE-AC02-05CH11231 (SM,CY), and by the Laboratory Directed Research and Development Program of Lawrence Berkeley National Laboratory under the U.S. Department of Energy contract number DEAC02-05CH11231 (A. S.), and by the Director, Office of Science, Advanced Scientific Computing Research, of the U.S. Department of Energy under Contract No. DEAC02-05CH11231 (F.M.). The computational results presented were obtained at the National Energy Research Scientific Computing Center (NERSC), which is supported by the Director, Office of Advanced Scientific Computing Research of the U.S. Department of Energy under contract number DE-AC02-05CH11232. Hau-tieng $\mathrm{Wu}$ acknowledges the support by Purdue National Science Foundation of United States (CCF-0939370) and Focused Research Group (DMS-1160319).

\section{Appendix A: Intensity fluctuations}

One of the practical issues one may face in ptychography is the intensity fluctuation among different diffraction frames introduced by instabilities in the light source, optics and shutters. Such fluctuation can be accounted for by introducing a scaling factor $\omega_{i} \in \mathbb{C}$ for each diffraction frame, As a result, the definition $\hat{z}_{i}$ is modified so that the equation

$$
\omega_{i} z_{(i)}=Q_{(i)} \psi
$$

holds for $i=1,2, \ldots, k$.

Since both $\omega_{i}$ and $\psi$ are unknown in (A1), the solution to (A1) is clearly not unique. To exclude the trivial solution $\omega_{i}=0$, for $i=1,2, \ldots k$ and $\psi=0$, we introduce an additional constraint and solve

$$
\begin{array}{r}
\left(\psi_{\min }, \omega_{(i)}\right)=\arg \min _{\psi, \omega_{(i)}} \sum_{i}\left\|Q_{(i)} \psi-\omega_{i} z_{(i)}\right\|^{2} \\
\text { subject to } \sum_{(i)} \omega_{i}=\sum_{(i)} 1=k,
\end{array}
$$

which is equivalent to solve

$$
\min _{\psi, \omega_{i}, \lambda} \mathcal{L}\left(\psi, \omega_{i}, \lambda\right), \text { where } \mathcal{L}=\sum_{i}\left\|Q_{(i)} \psi-\omega_{i} z_{(i)}\right\|^{2}+2 \lambda\left(\sum_{i} \omega_{i}-\|\mathbf{1}\|^{2}\right),
$$

where $\lambda$ is a Lagrange multiplier. To find the coefficients $\omega_{i}$, we use the normal equation associated with the LSQ problem (Eq. A3) :

$$
\left(\begin{array}{cccccc}
\sum_{i} Q_{(i)}^{*} Q_{(i)} & -Q_{1}^{*} z_{1} & \ldots & \ldots & -Q_{k}^{*} z_{k} & 0 \\
-z_{1}^{*} Q_{1} & z_{1}^{*} z_{1} & 0 & \ldots & 0 & 1 \\
\vdots & 0 & \ddots & & \vdots & \vdots \\
\vdots & \vdots & & & 0 & \\
-z_{k}^{*} Q_{k} & 0 & \ldots & 0 & z_{k}^{*} z_{k} & 1 \\
0 & 1 & \ldots & & 1 & 0
\end{array}\right)\left(\begin{array}{c}
\psi \\
\omega_{1} \\
\vdots \\
\omega_{k} \\
\lambda
\end{array}\right)=\left(\begin{array}{c}
0 \\
0 \\
\vdots \\
0 \\
\|\mathbf{1}\|^{2}
\end{array}\right)
$$

We can partition the equation above as

$$
\left(\begin{array}{ccc}
A & B^{*} & \mathbf{0} \\
B & D & \mathbf{1} \\
\mathbf{0} & \mathbf{1}^{*} & \mathbf{0}
\end{array}\right)\left(\begin{array}{c}
\psi \\
\boldsymbol{\omega} \\
\lambda
\end{array}\right)=\left(\begin{array}{c}
0 \\
0 \\
\|\mathbf{1}\|^{2}
\end{array}\right)
$$


where

$$
A=\sum_{i=1}^{k} Q_{(i)}^{*} Q_{(i)}, \quad B=\left(\begin{array}{c}
-z_{1}^{*} Q_{1} \\
\vdots \\
-z_{k}^{*} Q_{k}
\end{array}\right), \quad D=\left(\begin{array}{cccc}
z_{1}^{*} z_{1} & 0 & \ldots & 0 \\
0 & z_{2}^{*} z_{2} & \ddots & \vdots \\
\vdots & \ddots & \ddots & 0 \\
0 & \ldots & 0 & z_{k}^{*} z_{k}
\end{array}\right), \quad \boldsymbol{\omega}=\left(\begin{array}{c}
\omega_{1} \\
\vdots \\
\omega_{k}
\end{array}\right)
$$

By the block factorization

$$
\left(\begin{array}{ccc}
I & 0 & 0 \\
-B A^{-1} & I & 0 \\
0 & -\mathbf{1}^{*} H^{-1} & 1
\end{array}\right)\left(\begin{array}{ccc}
A & B^{*} & \mathbf{0} \\
\mathbf{0} & H & \mathbf{1} \\
\mathbf{0} & \mathbf{0} & -\mathbf{1}^{*} H^{-1} \mathbf{1}
\end{array}\right)\left(\begin{array}{c}
\psi \\
\boldsymbol{\omega} \\
\lambda
\end{array}\right)=\left(\begin{array}{c}
0 \\
0 \\
\|\mathbf{1}\|^{2}
\end{array}\right),
$$

where the Schur complement $H=D-B A^{-1} B^{*}$ is given by

$$
H_{i, j}=z_{(i)}^{*}\left(\delta_{i, j} I-Q_{(i)}\left(\sum_{(\iota)} Q_{(\iota)}^{*} Q_{(\iota)}\right)^{-1} Q_{(j)}^{*}\right) z_{(j)} .
$$

By block-wise inversion of Eq. A4, we obtain $\boldsymbol{\omega}$ and the scaling factor $\lambda$ from a sparse linear equation:

$$
H \boldsymbol{\omega}=-\lambda \mathbf{1}, \quad \lambda=-\frac{\|\mathbf{1}\|^{2}}{\mathbf{1}^{*} H^{-1} \mathbf{1}} .
$$

\section{Eigenvalue method}

If we make a change of variable, $\nu_{(i)}=\left\|z_{(i)}\right\| \omega_{(i)}$, we can re-write the problem $\arg \min _{\boldsymbol{\omega}} \boldsymbol{\omega}^{*} H \boldsymbol{\omega}$ as:

$$
\arg \min _{\boldsymbol{\nu}}\|\boldsymbol{\nu}\|^{2}-\boldsymbol{\nu}^{*} K \boldsymbol{\nu}, \quad K_{i, j}=\frac{z_{(i)}^{*}}{\left\|z_{(i)}\right\|} Q_{(i)}\left(\sum_{(\iota)} Q_{(\iota)}^{*} Q_{(\iota)}\right)^{-1} Q_{(j)}^{*} \frac{z_{(j)}}{\left\|z_{(j)}\right\|} .
$$

The solution to this problem assuming $\|\nu\|=$ constant is the eigenvector corresponding to the largest eigenvalue of the sparse matrix $K$. This can be computed efficiently using packages such as ${ }^{41}$

\section{Appendix B: Taylor expansion}

We consider the case in which the probe $w$ is translated from the input coordinate by an unknown distance $\xi$. We restrict ourselves to the 1-dim ptychography problem to simplify the discussion. We call $\boldsymbol{Q}_{\xi}$ the unknown illumination matrix used to generate the data. To determine the illumination matrix, we determine the parameter $\xi$ so that the error $\varepsilon_{\boldsymbol{Q}_{\xi}}$ is minimized:

$$
\arg \min _{\xi \in \mathbb{R}^{k}}\left\|\left[I-P_{\boldsymbol{Q}_{\xi}}\right] \boldsymbol{z}\right\|^{2},
$$

where the $i$-th entry of $\xi \in \mathbb{R}^{k}$ represents the translation distance of the $i$-th frame. Given the illumination function $w$, we can compute the first and second order derivatives with respect to translation. We denote by $Q_{i}, R_{i}, S_{i}$ the illumination matrices that extract a frame out of an image and multiplies by $\left(w(\boldsymbol{x}), \partial_{x} w(\boldsymbol{x}), \partial_{x}^{2} w(\boldsymbol{x})\right)$ respectively. Build $\boldsymbol{Q}, \boldsymbol{R}, \boldsymbol{S}$ from $Q_{i}, R_{i}, S_{i}$, which are tall and skinny matrices of the same size as $\boldsymbol{Q}$ discussed earlier, with identical location of the non-zero entries. Assume that the probe is perturbed to second order as follows:

$$
\boldsymbol{Q}_{\xi}=\boldsymbol{Q}+\xi \boldsymbol{R}+\xi^{2} \boldsymbol{S} .
$$

where, by a slight abuse of notation, $\xi$ denotes a diagonal and real matrix so that the $i$-th diagonal entry, denoted as $\xi_{i}$ represents the translation distance of the $i$-th frame. With $\boldsymbol{Q}_{\xi}$ plugged into (B1), we now minimize

$$
\arg \min _{\xi \in \mathbb{R}^{k}}\left\|\left[I-\left(\boldsymbol{Q}+\xi \boldsymbol{R}+\xi^{2} \boldsymbol{S}\right)\left[\left(\boldsymbol{Q}+\xi \boldsymbol{R}+\xi^{2} \boldsymbol{S}\right)^{*}\left(\boldsymbol{Q}+\xi \boldsymbol{R}+\xi^{2} \boldsymbol{S}\right)\right]^{-1}\left(\boldsymbol{Q}+\xi \boldsymbol{R}+\xi^{2} \boldsymbol{S}\right)^{*}\right] \boldsymbol{z}\right\|^{2} .
$$


By Taylor expansion:

$$
[\cdot]^{-1} \simeq \frac{1}{\boldsymbol{Q}^{*} \boldsymbol{Q}}\left(1-\left(\boldsymbol{R}^{*} \xi \boldsymbol{Q}+\boldsymbol{Q}^{*} \xi \boldsymbol{R}+O\left(\xi^{2}\right)\right) \frac{1}{\boldsymbol{Q}^{*} \boldsymbol{Q}}\right)
$$

when $\boldsymbol{Q}^{*} \boldsymbol{Q}$ is invertible. The second order term $O\left(\xi^{2}\right)$ includes other second order terms that we will not need. We write the expansion of the residual in Eq. (B2) $f_{0}+f_{1}(\xi)+f_{2}\left(\xi^{2}\right)$ as:

$$
f_{0}=\left[I-P_{Q}\right] z \equiv \underline{z}
$$

We define $\phi^{*} \equiv \frac{1}{\boldsymbol{Q}^{*} \boldsymbol{Q}} \boldsymbol{Q}^{*}$, and express the first order as

$$
f_{1}(\xi)=\left[-\xi \boldsymbol{R} \phi^{*}-\phi \boldsymbol{R}^{*} \xi+\phi\left(\boldsymbol{R}^{*} \xi \boldsymbol{Q}+\boldsymbol{Q}^{*} \xi \boldsymbol{R}\right) \phi^{*}\right] \boldsymbol{z} .
$$

By defining $\boldsymbol{z}_{\boldsymbol{R}} \equiv \boldsymbol{R} \phi^{*} \boldsymbol{z}$, using $P_{\boldsymbol{Q}}=\boldsymbol{Q} \phi^{*}=\phi \boldsymbol{Q}^{*}$, and rearranging, we get

$$
\begin{aligned}
f_{1}(\xi) & =-\xi \boldsymbol{z}_{\boldsymbol{R}}-\phi \boldsymbol{R}^{*} \xi\left(\boldsymbol{z}-\boldsymbol{Q} \phi^{*} \boldsymbol{z}\right)+\phi \boldsymbol{Q}^{*} \xi \boldsymbol{z}_{\boldsymbol{R}} \\
& =-\left(1-P_{\boldsymbol{Q}}\right) \xi \boldsymbol{z}_{\boldsymbol{R}}-\phi \boldsymbol{R}^{*} \xi \underline{\boldsymbol{z}} .
\end{aligned}
$$

By using the equality $\underline{\boldsymbol{z}}\left(I-P_{Q}\right)=\underline{\boldsymbol{z}}$, setting $O_{\boldsymbol{R}} \equiv \boldsymbol{R} \phi^{*} \phi \boldsymbol{R}^{*}=\boldsymbol{R} \frac{1}{\boldsymbol{Q}^{*} \boldsymbol{Q}} \boldsymbol{R}^{*}$ and rearranging, we obtain:

$$
\begin{aligned}
f_{2}(\xi) & =-\left[\xi \boldsymbol{R}_{\frac{1}{\boldsymbol{Q}^{*} \boldsymbol{Q}}} \boldsymbol{R}^{*} \xi+\xi^{2} \boldsymbol{S} \phi+\boldsymbol{Q O}\left(\xi^{2}\right)\right] \boldsymbol{z} \\
& =-\xi O_{\boldsymbol{R}} \xi \boldsymbol{z}-\xi^{2} \boldsymbol{z}_{\boldsymbol{S}}+\boldsymbol{Q} O\left(\xi^{2}\right) \boldsymbol{z}
\end{aligned}
$$

where $\boldsymbol{z}_{\boldsymbol{S}} \equiv \boldsymbol{S} \phi^{*} \boldsymbol{z}$. We rewrite Eq. (B2) above as:

$$
\left\|\left[I-P_{Q_{\xi}}\right] \boldsymbol{z}\right\|^{2}=f_{0}^{*} f_{0}+f_{0}^{*} f_{1}+f_{1}^{*} f_{0}+f_{1}^{*} f_{1}+f_{0}^{*} f_{2}+f_{2}^{*} f_{0}+O\left(\xi^{3}\right) .
$$

Note that $\underline{\boldsymbol{z}}^{*} \boldsymbol{Q}=\underline{\boldsymbol{z}}^{*} \phi=0$. Set $\boldsymbol{z}_{\boldsymbol{Q}} \equiv \boldsymbol{Q} \phi \boldsymbol{z}$ and obtain the first and second order terms of Eq. (B2):

$$
\begin{aligned}
f_{0}^{*} f_{1}+f_{1}^{*} f_{0} & =-\underline{\boldsymbol{z}}^{*} \xi \boldsymbol{z}_{\boldsymbol{R}},-\boldsymbol{z}_{\boldsymbol{R}}^{*} \xi \underline{\boldsymbol{z}} \\
f_{1}^{*} f_{1}+f_{0}^{*} f_{2}+f_{2}^{*} f_{0} & =\boldsymbol{z}_{\boldsymbol{R}}^{*} \xi\left(I-P_{\boldsymbol{Q}}\right) \xi \boldsymbol{z}_{\boldsymbol{R}}+\underline{\boldsymbol{z}} \xi O_{\boldsymbol{R}} \xi \underline{\boldsymbol{z}}-\underline{\boldsymbol{z}}^{*} \xi O_{\boldsymbol{R}} \xi \boldsymbol{z}-\underline{\boldsymbol{z}}^{*} \xi^{2} \boldsymbol{z}_{\boldsymbol{S}}-\boldsymbol{z}^{*} \xi O_{\boldsymbol{R}} \xi \underline{\boldsymbol{z}}-\boldsymbol{z}_{\boldsymbol{S}}^{*} \xi^{2} \underline{\boldsymbol{z}}, \\
& =\boldsymbol{z}_{\boldsymbol{R}}^{*} \xi^{2} \boldsymbol{z}_{\boldsymbol{R}}-\underline{\boldsymbol{z}}^{*} \xi^{2} \boldsymbol{z}_{\boldsymbol{S}}-\boldsymbol{z}_{\boldsymbol{S}}^{*} \xi^{2} \underline{\boldsymbol{z}}-\boldsymbol{z}^{*} \xi O_{\boldsymbol{R}} \xi \boldsymbol{z}+\boldsymbol{z}_{\boldsymbol{Q}}^{*} \xi O_{\boldsymbol{R}} \xi \boldsymbol{z}_{\boldsymbol{Q}}-\boldsymbol{z}_{\boldsymbol{R}}^{*} \xi P_{\boldsymbol{Q}} \xi \boldsymbol{z}_{\boldsymbol{R}},
\end{aligned}
$$

By using the definition of $\boldsymbol{z}_{\boldsymbol{Q}}, \boldsymbol{z}_{\boldsymbol{R}}, P_{\boldsymbol{Q}}$ and $O_{\boldsymbol{R}}$ it is easy to show that $\boldsymbol{z}_{\boldsymbol{Q}}^{*} \xi O_{\boldsymbol{R}} \xi \boldsymbol{z}_{\boldsymbol{Q}}=\boldsymbol{z}_{\boldsymbol{R}}^{*} \xi P_{\boldsymbol{Q}} \xi \boldsymbol{z}_{\boldsymbol{R}}$ and simplify as:

$$
f_{1}^{*} f_{1}+f_{0}^{*} f_{2}+f_{2}^{*} f_{0}=z_{\boldsymbol{R}}^{*} \xi^{2} z_{\boldsymbol{R}}-\underline{\boldsymbol{z}}^{*} \xi^{2} \boldsymbol{z}_{\boldsymbol{S}}-\boldsymbol{z}_{\boldsymbol{S}}^{*} \xi^{2} \underline{\boldsymbol{z}}-\boldsymbol{z}^{*} \xi O_{\boldsymbol{R}} \xi \boldsymbol{z}
$$

By setting $\partial_{\xi_{i}}\|\cdot\|^{2}=0$ in Eq. (B2), we obtain the linear equation for solving $\xi$ :

$$
\sum_{j}\left(2\left(\boldsymbol{z}_{\boldsymbol{R}_{i}}^{*} \boldsymbol{z}_{\boldsymbol{R}_{i}}-\underline{\boldsymbol{z}}_{i}^{*} \boldsymbol{z}_{\boldsymbol{S}_{i}}-\boldsymbol{z}_{\boldsymbol{S}_{i}}^{*} \underline{\boldsymbol{z}}_{i}\right) \delta_{i j}-\boldsymbol{z}_{i}^{*} O_{\boldsymbol{R}_{i j}} \boldsymbol{z}_{j}-\boldsymbol{z}_{j}^{*} O_{\boldsymbol{R}_{j i}} \boldsymbol{z}_{i}\right) \xi_{j}=\underline{\boldsymbol{z}}_{i}^{*} \boldsymbol{z}_{\boldsymbol{R}_{i}}+\boldsymbol{z}_{\boldsymbol{R}_{i}}^{*} \underline{\boldsymbol{z}}_{i}
$$

We note that $-\underline{\boldsymbol{z}}^{*} \xi^{2} \boldsymbol{z}_{\boldsymbol{S}}-\boldsymbol{z}_{\boldsymbol{S}}^{*} \xi^{2} \underline{\boldsymbol{z}}$ is a second order correction if we assume that $\boldsymbol{z}$ is in that range of an unknown $\boldsymbol{Q}_{\xi}$ for small $\xi$. We thus have the following approximation:

$$
f_{1}^{*} f_{1}+f_{0}^{*} f_{2}+f_{2}^{*} f_{0} \simeq \boldsymbol{z}_{\boldsymbol{R}}^{*} \xi^{2} \boldsymbol{z}_{\boldsymbol{R}}-\boldsymbol{z}^{*} \xi O_{\boldsymbol{R}} \xi \boldsymbol{z} .
$$

We can thus consider solving the following approximation equation in practice:

$$
\sum_{j}\left(2 \boldsymbol{z}_{\boldsymbol{R}_{i}}^{*} \boldsymbol{z}_{\boldsymbol{R}_{i}} \delta_{i j}-\boldsymbol{z}_{i}^{*} O_{\boldsymbol{R}_{i j}} \boldsymbol{z}_{j}-\boldsymbol{z}_{j}^{*} O_{\boldsymbol{R}_{j i}} \boldsymbol{z}_{i}\right) \xi_{j} \simeq \underline{\boldsymbol{z}}_{i}^{*} \boldsymbol{z}_{\boldsymbol{R}_{i}}+\boldsymbol{z}_{\boldsymbol{R}_{i}}^{*} \underline{\boldsymbol{z}}_{i}
$$

Extension to the two dimensional case is given in Section IV. 


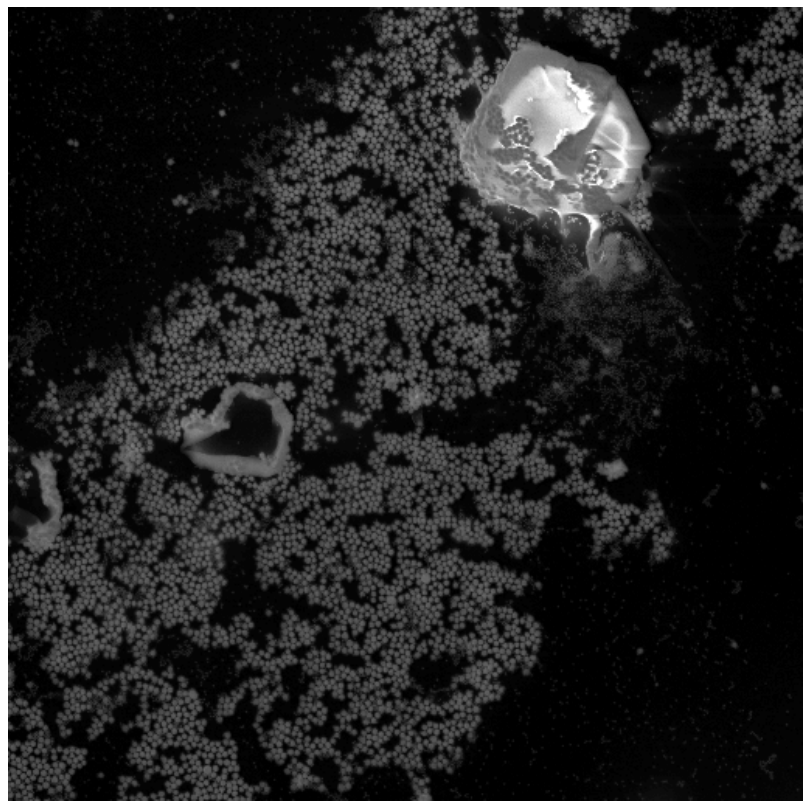

FIG. 2: object $\psi$ used to simulate diffraction data)

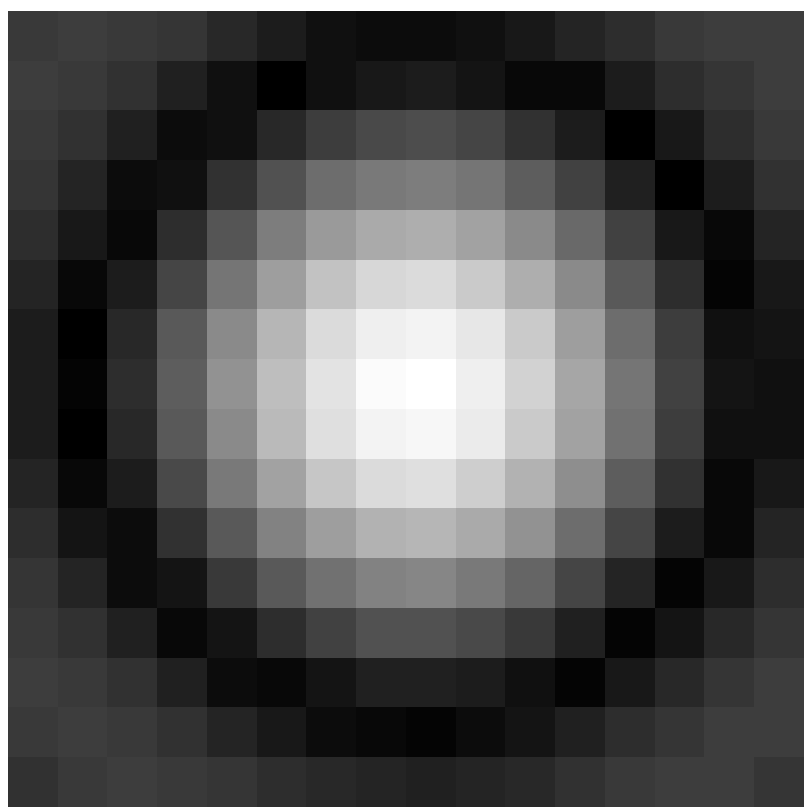

FIG. 3: Absolute value of the probe $|w(\boldsymbol{r})|$ used in simulations $(16 \times 16$ pixels $)$

* smarchesini@lbl.gov

† cyang@lbl.gov

‡ hauwu@berkeley.edu

$\S$ frmaia@lbl.gov

1 W. Hoppe. Beugung im inhomogenen Primärstrahlwellenfeld. I. Prinzip einer Phasenmessung von Elektronenbeungungsinterferenzen. Acta Crystallographica Section A, 25(4):495-501, Jul 1969.

2 R. Hegerl and W. Hoppe. Dynamic theory of crystalline structure analysis by electron diffraction in inhomogeneous primary wave field. Berichte Der Bunsen-Gesellschaft Fur Physikalische Chemie, 74:1148, 1970.

3 J. M. Rodenburg and R. H. T. Bates. The theory of super-resolution electron microscopy via wigner-distribution deconvolution. Phil. Trans. R. Soc. Lond. A, 339:521-553, 1992.

4 John CH Spence. High-resolution electron microscopy, volume 60. Clarendon Press, 2003.

${ }^{5}$ Ch. Broennimann, E. F. Eikenberry, B. Henrich, R. Horisberger, G. Huelsen, E. Pohl, B. Schmitt, C. Schulze-Briese, M. Suzuki, T. Tomizaki, H. Toyokawa, and A. Wagner. The pilatus $1 \mathrm{~m}$ detector. Journal of Synchrotron Radiation, 13(2):120-130, 2006.

${ }^{6}$ D. Doering, Y.-D. Chuang, N. Andresen, K. Chow, D. Contarato, C. Cummings, E. Domning, J. Joseph, J. S. Pepper, B. Smith, G. Zizka, C. Ford, W. S. Lee, M. Weaver, L. Patthey, J. Weizeorick, Z. Hussain, and P. Denes. Development of a compact fast ccd camera and resonant soft x-ray scattering endstation for time-resolved pump-probe experiments. Review of Scientific Instruments, 82(7):073303, 2011.

7 H. N. Chapman. Phase-retrieval x-ray microscopy by wigner -distribution deconvolution. Ultramicroscopy, 66:153-172, 1996.

8 P. Thibault, M. Dierolf, A. Menzel, O. Bunk, C. David, and F. Pfeiffer. High-Resolution scanning x-ray diffraction microscopy. Science, 321(5887):379-382, 2008.

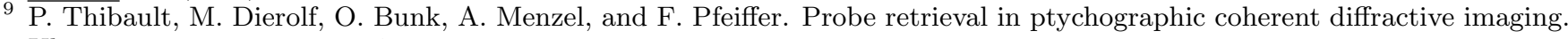
Ultramicroscopy, 109:338-343, 2009.

10 J. M. Rodenburg and H. M. L. Faulkner. A phase retrieval algorithm for shifting illumination. Appl. Phy. Lett., 85:4795-4797, 2004.

11 C.M. Kewish, P. Thibault, M. Dierolf, O. Bunk, A. Menzel, J. Vila-Comamala, K. Jefimovs, and F. Pfeiffer. Ptychographic characterization of the wavefield in the focus of reflective hard x-ray optics. Ultramicroscopy, 110:325-9, Mar 2010.

12 Susanne Hönig, Robert Hoppe, Jens Patommel, Andreas Schropp, Sandra Stephan, Sebastian Schöder, Manfred Burghammer, and Christian G. Schroer. Full optical characterization of coherent x-ray nanobeams by ptychographic imaging. Opt. Express, 19(17):16324-16329, Aug 2011.

13 Manuel Guizar-Sicairos, Suresh Narayanan, Aaron Stein, Meredith Metzler, Alec R. Sandy, James R. Fienup, and Kenneth Evans-Lutterodt. Measurement of hard x-ray lens wavefront aberrations using phase retrieval. Applied Physics Letters, 98(11):111108, 2011. 

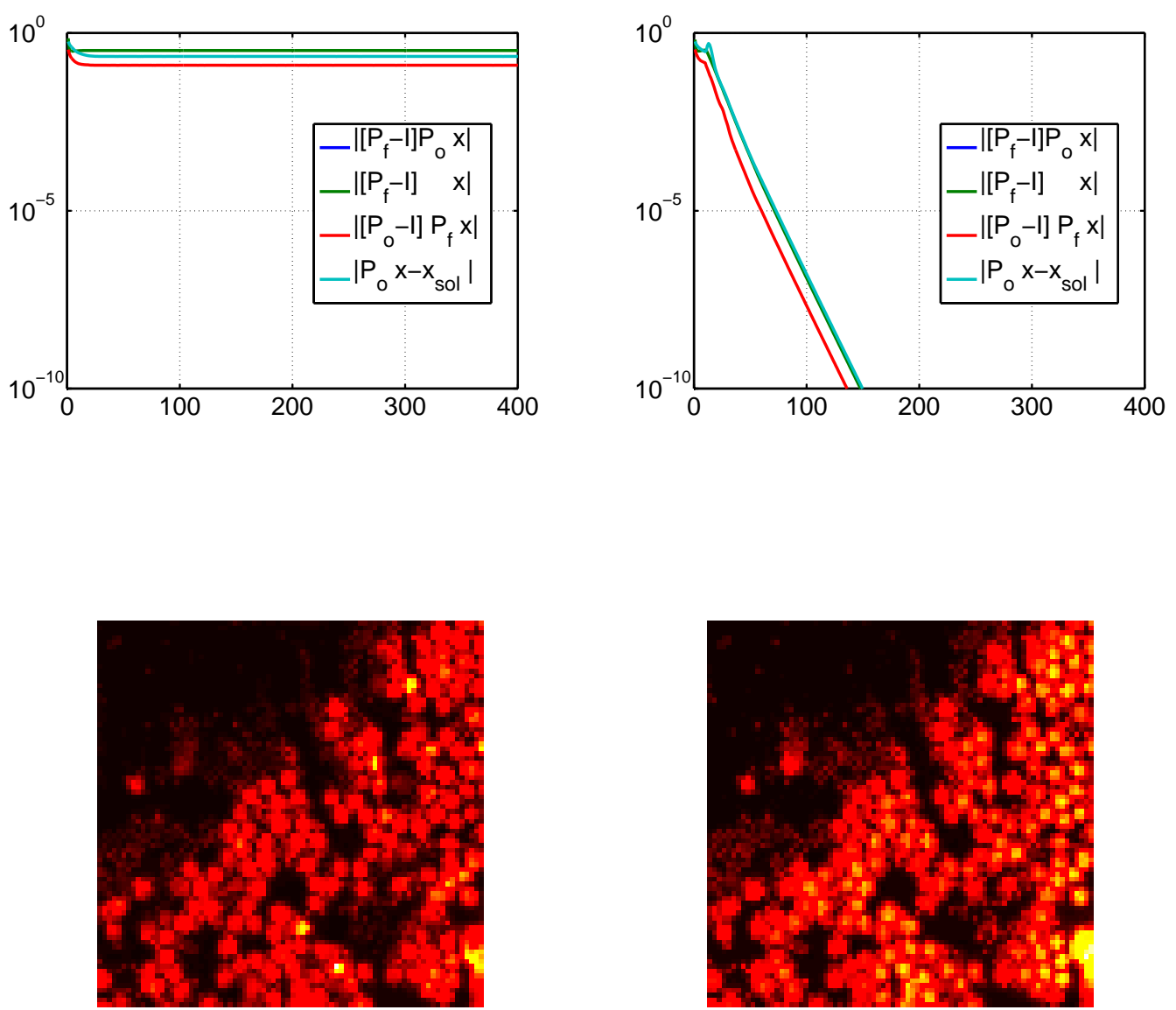

FIG. 4: Convergence rate with an I0-error of $\pm 20 \%$. (left) old projection operator (right) new projection operator. (bottom) reconstruction from data with I0-error, and solution (reconstruction using the new projection operator is within the computer numerical precision, i.e. the figure on the right looks identical to the exact solution.

14 M. Guizar-Sicairos and J. R. Fienup. Phase retrieval with transverse translation diversity: a nonlinear optimization approach. Opt. Express, 16:7264-7278, 2008.

15 A.M. Maiden, M.J. Humphry, M.C. Sarahan, B. Kraus, and J.M. Rodenburg. An annealing algorithm to correct positioning errors in ptychography. Ultramicroscopy, 120(0):64 - 72, 2012.

16 Mike Beckers, Tobias Senkbeil, Thomas Gorniak, Klaus Giewekemeyer, Tim Salditt, and Axel Rosenhahn. Drift correction in ptychographic diffractive imaging. Ultramicroscopy, 126(0):44 - 47, 2013.

17 Samuel T Thurman and James R Fienup. Phase retrieval with signal bias. JOSA A, 26(4):1008-1014, 2009.

18 Manuel Guizar-Sicairos and James R. Fienup. Measurement of coherent x-ray focusedbeams by phase retrieval with transversetranslation diversity. Opt. Express, 17(4):2670-2685, Feb 2009.

19 P. Thibault and M. Guizar-Sicairos. Maximum-likelihood refinement for coherent diffractive imaging. New Journal of Physics, 14(6):063004, 2012.

20 Pierre Godard, Marc Allain, Virginie Chamard, and John Rodenburg. Noise models for low counting rate coherent diffraction imaging. Opt. Express, 20(23):25914-25934, Nov 2012.

21 N. C. Jesse and G. P. Andrew. Simultaneous sample and spatial coherence characterisation using diffractive imaging. Applied Physics Letters, 99(15):154103, 2011.

22 J. R. Fienup, J. C. Marron, T. J. Schulz, and J. H. Seldin. Hubble space telescope characterized by using phase-retrieval algorithms. Appl. Opt., 32(10):1747-1767, Apr 1993.

23 Brian Abbey, Keith A. Nugent, Garth J. Williams, Jesse N. Clark, Andrew G. Peele, Mark A. Pfeifer, Martin de Jonge, and Ian McNulty. Keyhole coherent diffractive imaging. Nature Physics, 4:394-398, 032008. 


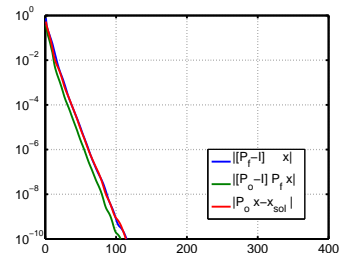

(a) $8 \times 8$ frames

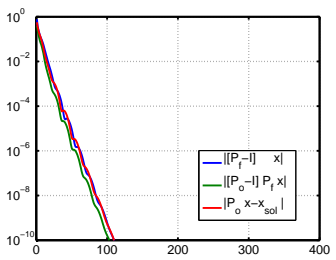

(e) $8 \times 8$ frames

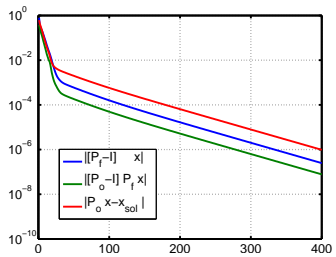

(b) $16 \times 16$ frames

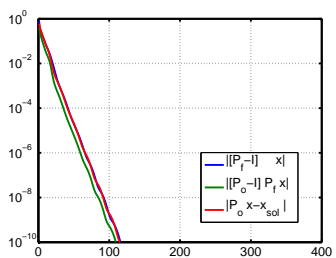

(f) $16 \times 16$ frames
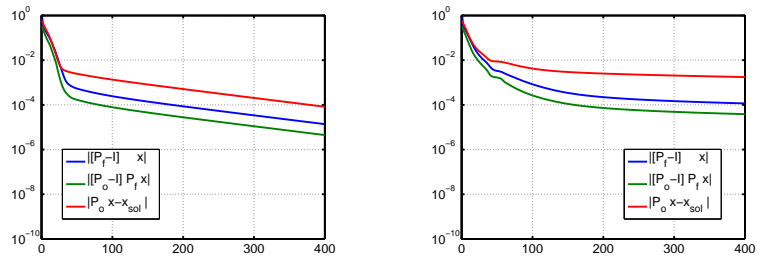

(c) $32 \times 32$ frames

(d) $64 \times 64$ frames
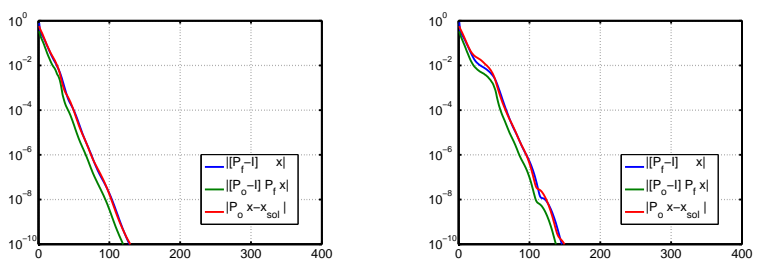

(h) $64 \times 64$ frames

FIG. 5: Convergence rate $\left(\varepsilon_{F}, \varepsilon_{Q}, \varepsilon_{0}\right.$ vs number of iteration $\ell$ ) for (top) regular reconstruction. (bottom) using augmented projection $\left(m=16\right.$ and step size $\left.x_{1}-x_{2}=3\right)$

${ }^{24}$ L. W. Whitehead, G. J. Williams, H. M. Quiney, D. J. Vine, R. A. Dilanian, S. Flewett, K. A. Nugent, A. G. Peele, E. Balaur, and I. McNulty. Diffractive imaging using partially coherent x rays. Phys. Rev. Lett., 103:243902, Dec 2009.

25 Jinsong $\mathrm{Wu}$, Kurt Leinenweber, John CH Spence, and Michael O'Keeffe. Ab initio phasing of x-ray powder diffraction patterns by charge flipping. Nature materials, 5(8):647-652, 2006.

26 J. M. Rodenburg. Ptychography and related diffractive imaging methods. Advances in Imaging and Electron Physics, 150, 2008.

27 H. M. L. Faulkner and J. M. Rodenburg. Movable aperture lensless transmission microscopy: a novel phase retrieval algorithm. Phy. Rev. Lett., 93:023903, 2004.

28 M. Dierolf, A. Menzel, P. Thibault, P. Schneider, C. M. Kewish, R. Wepf, O. Bunk, and F. Pfeiffer. Ptychographic x-ray computed tomography at the nanoscale. Nature, 467:436-439, 2011.

29 Z. Wen, C. Yang, X. Liu, and S. Marchesini. Alternating direction methods for classical and ptychographic phase retrieval. Inverse Problems, 28(11):115010, 2012.

30 C. Yang, J. Qian, A. Schirotzek, F. Maia, and S. Marchesini. Iterative algorithms for ptychographic phase retrieval. Technical Report 4598E, arXiv:1105.5628, Lawrence Berkeley National Laboratory, 2011.

31 J. Qian, C. Yang, A. Schirotzek, F. Maia, and S. Marchesini. Iefficient algorithms for ptychographic phase retrieval. Contemporary Mathematics, to appear, 2013.

32 E. J. Candes, T. Strohmer, and V. Voroninski. Phaselift: Exact and stable signal recovery from magnitude measurements via convex programming. Communications on Pure and Applied Mathematics, 66(8):1241-1274, 2013.

${ }^{33}$ H. Ohlsson, A. Y. Yang, R. Dong, and S. Shankar Sastry. Compressive phase retrieval from squared output measurements via semidefinite programming. IEEE Conference on Decision and Control, CDC 2012, November 2011.

${ }^{34}$ E. J. Candes, Y. Eldar, T. Strohmer, and V. Voroninski. Phase retrieval via matrix completion. ArXiv e-prints, September 2011.

${ }^{35}$ I. Waldspurger, A. d'Aspremont, and S. Mallat. Phase recovery, MaxCut and complex semidefinite programming. ArXiv, June 2012.

36 Zaiwen Wen, Donald Goldfarb, and Katya Scheinberg. Block coordinate descent methods for semidefinite programming. In Miguel F. Anjos and Jean B. Lasserre, editors, Handbook on Semidefinite, Conic and Polynomial Optimization, volume 166 of International Series in Operations Research \& Management Science, pages 533-564. Springer US, 2012. 

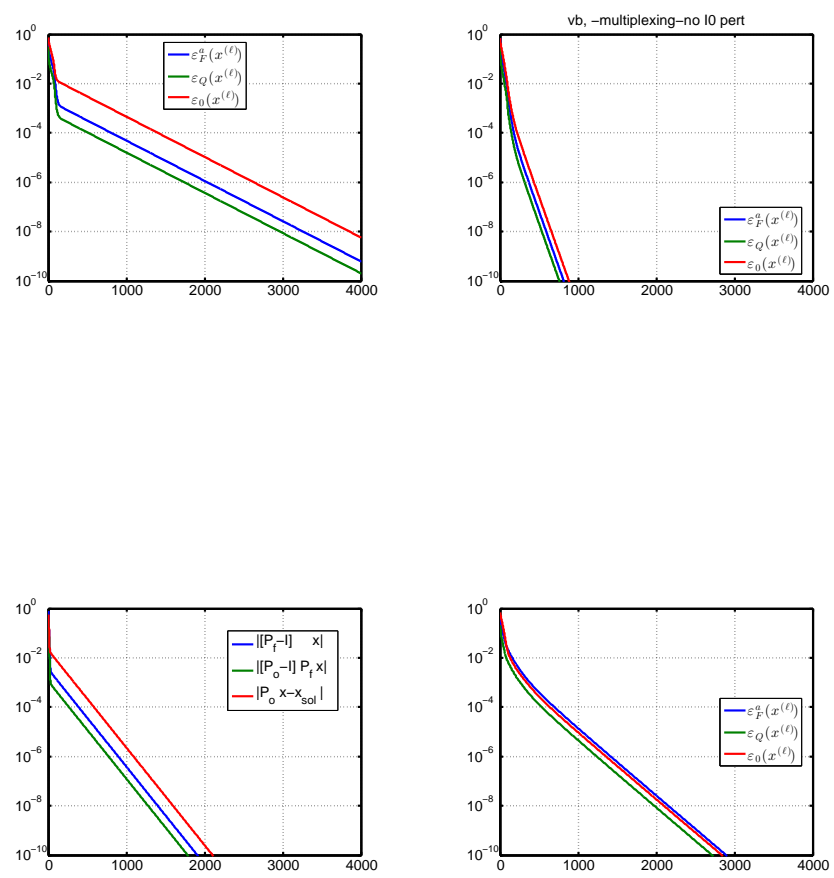

FIG. 6: Convergence rate with incoherent illumination of 4 beams, separated by $3 \times$ the probe width (FWHM) using standard projection algorithms (top left), with intermediate phase optimization (top-right), phase and amplitude(bottom-left), and phase and amplitude with initial amplitude error of $20 \%$ (bottom right), frame width $16 \times 16,16 \times 16$ frames, step size 3.5 pixels close packing with \pm 1 pixel known random perturbations.

37 J. R. Fienup. Phase retrieval algorithms: a comparison. Appl. Opt., 21:2758-2769, 1982.

${ }^{38}$ R. Luke. Relaxed averaged alternating reflections for diffraction imaging. Inverse Problems, 21:37-50, 2005.

39 A. Singer. Angular synchronization by eigenvectors and semidefinite programming. Applied and Computational Harmonic Analysis, 30(1):20 - 36, 2011.

40 Pierre Thibault and Andreas Menzel. Reconstructing state mixtures from diffraction measurements. Nature, 494(7435):6871, 2013.

41 R. B. Lehoucq, D. C. Sorensen, and C. Yang. Arpack users guide: Solution of large scale eigenvalue problems by implicitly restarted arnoldi methods., 1997. 


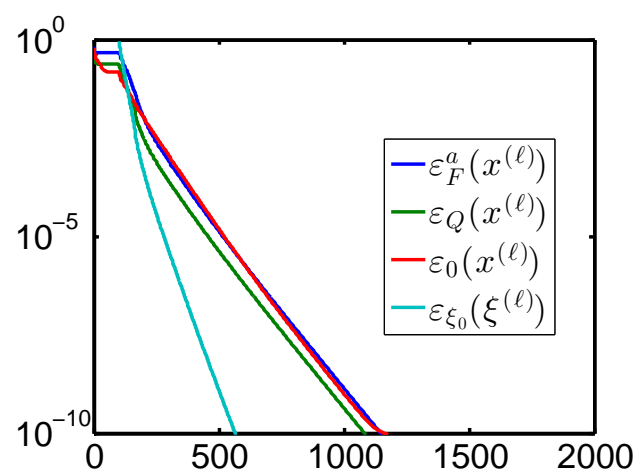

FIG. 7: Reconstruction with position errors using the method described in section IV, where $\varepsilon_{\xi_{0}}=\left\|\xi-\xi_{0}\right\| /\left\|\xi_{0}\right\|$, and the perturbations in position are randomly distributed with $\left\langle\xi_{0}\right\rangle=\frac{1}{k} \sum_{i}\left\|\xi_{i}\right\|=2.5$ resolution elements.

(number of frames: $16 \times 16$, frame dimensions $32 \times 32$, step size: 3.5 pixels, hexagonal packing with known random perturbations of \pm 1 pixels and unknown $\xi$ random perturbations ).
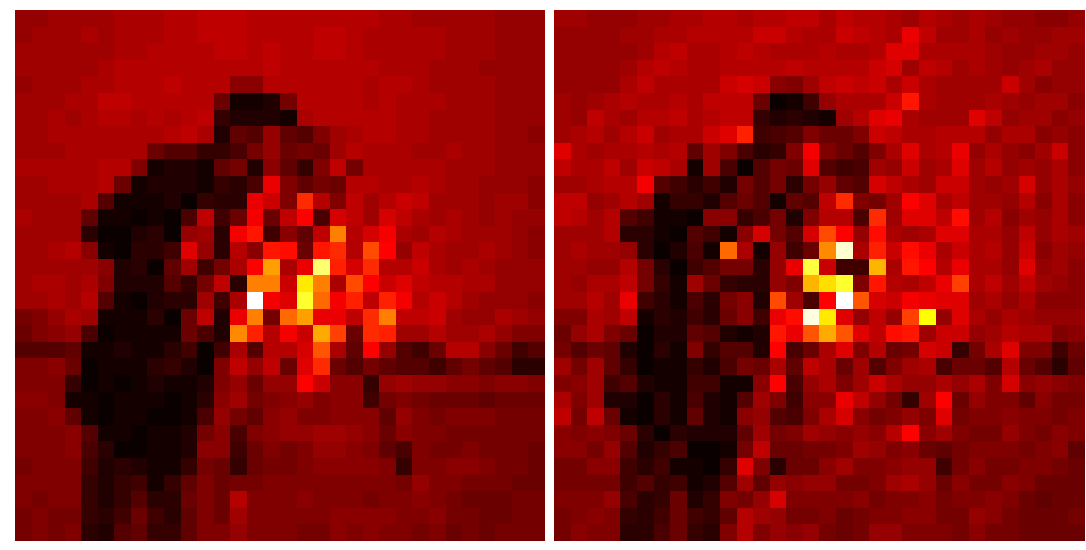

FIG. 8: Two measured intensities with additive background $(\mathrm{SNR}=0.5)$. In a separate test the diffraction data was buried by the background ( (in other figures not included background was $10^{6} \times$ the signal). 

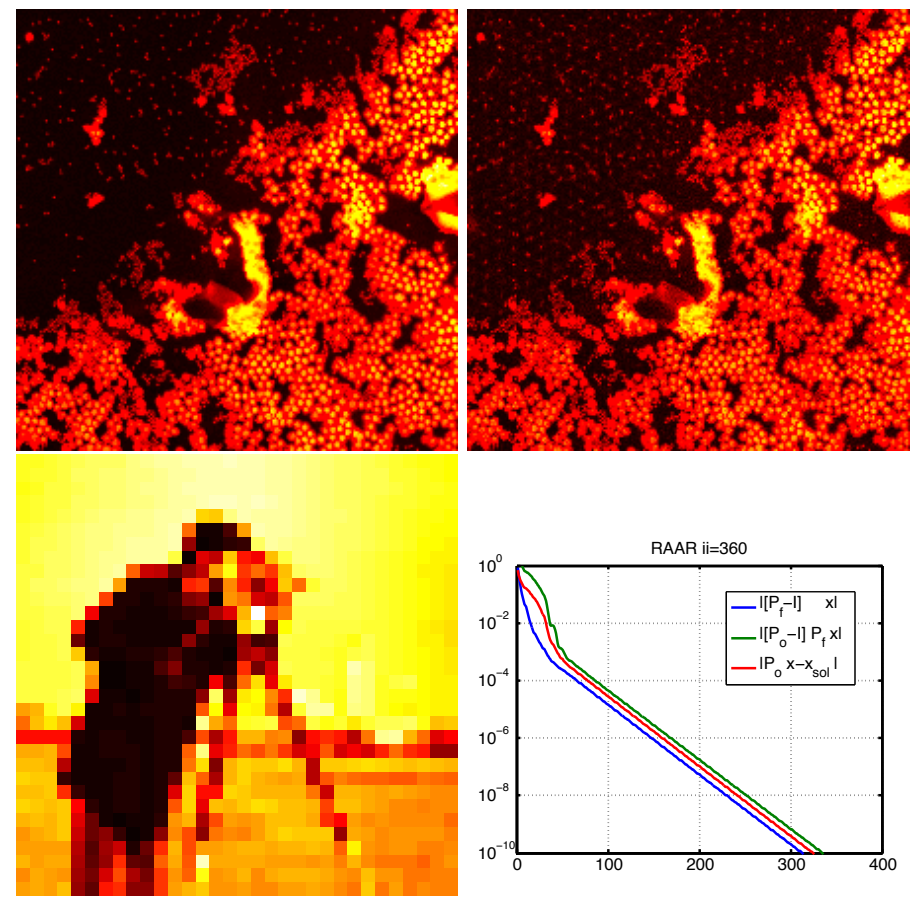

FIG. 9: (top) reconstructed image with background optimization (left) and without (right). The figure on the left looks identical to the exact solution. (bottom) reconstructed background (left), convergence behavior(right). 

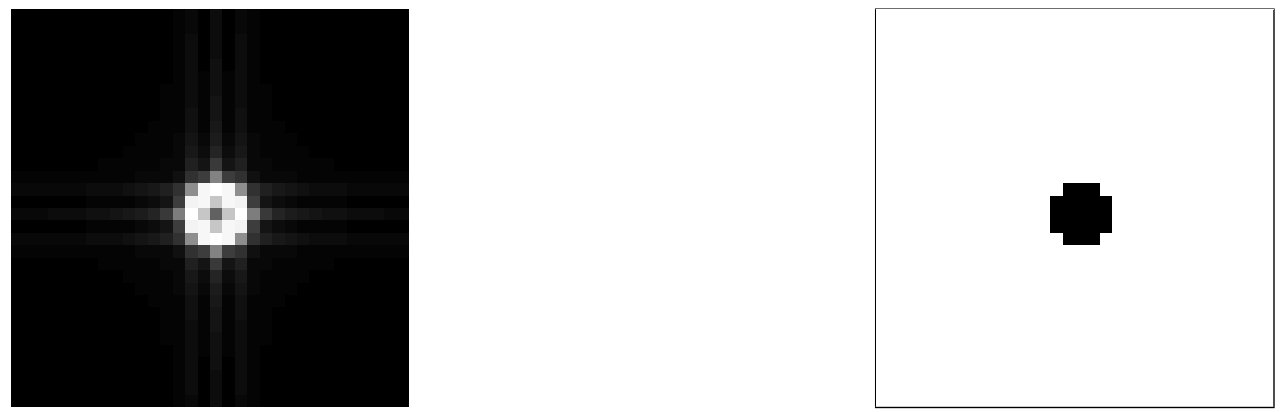

FIG. 10: Fourier transform of the probe $w$ used to simulate the experiment

FIG. 11: Map of $\frac{1}{1+\sigma^{2}}$. The $\sigma$ are $\infty$ on dark pixels and 0 on white pixels.

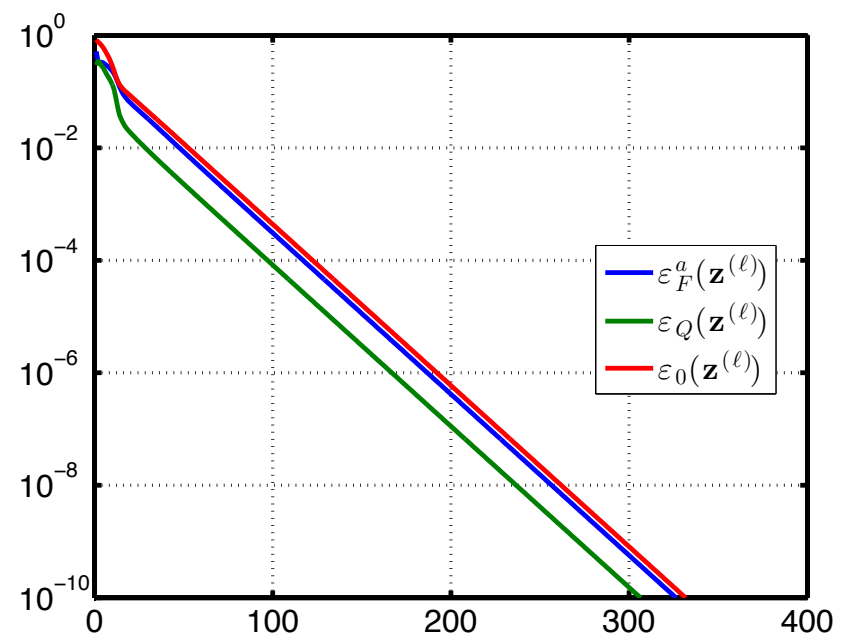

FIG. 12: Convergence behavior with missing data. Frame size: $32 \times 32$, number of frames: $16 \times 16$, step size: 3.5 pixels 